\title{
A Hybrid Model for Pricing and Hedging of Long Dated Bonds
}

This version: 24 April 2015

\author{
Jan Baldeaux ${ }^{\mathrm{a}}$, Man Chung Fung ${ }^{\mathrm{b}}$, Katja Ignatieva ${ }^{\mathrm{c}}$, Eckhard Platen ${ }^{\mathrm{d}}$ \\ ${ }^{a}$ Finance Discipline Group, Business School, University of Technology Sydney, Australia \\ (jan.baldeaux@uts.edu.au) \\ ${ }^{\mathrm{b}}$ School of Risk and Actuarial Studies, Business School, University of New South Wales Sydney, Australia \\ (m.c.fung@student.unsw.edu.au) \\ ${ }^{\mathrm{c}}$ School of Risk and Actuarial Studies, Business School, University of New South Wales Sydney, Australia \\ (k.ignatieva@unsw.edu.au) \\ ${ }^{\mathrm{d}}$ Quantitative Finance Research Centre, Finance Discipline Group, Business School, University of \\ Technology Sydney Australia (eckhard.platen@uts.edu.au)
}

\begin{abstract}
Long dated fixed income securities play an important role in asset-liability management, in life insurance and in annuity businesses. This paper applies the benchmark approach, where the growth optimal portfolio (GOP) is employed as numéraire together with the real world probability measure for pricing and hedging of long dated bonds. It employs a time dependent constant elasticity of variance model for the discounted GOP and takes stochastic interest rate risk into account. This results in a hybrid framework that models the stochastic dynamics of the GOP and the short rate simultaneously. We estimate and compare a variety of continuous-time models for short-term interest rates using non-parametric kernel-based estimation. The hybrid models remain highly tractable and fit reasonably well the observed dynamics of proxies of the GOP and interest rates. Our results involve closed-form expressions for bond prices and hedge ratios. Across all models under consideration we find that the hybrid model with the $3 / 2$ dynamics for the interest rate provides the best fit to the data with respect to lowest prices and least expensive hedges.
\end{abstract}

Key words: Long dated bond pricing, stochastic interest rate, growth optimal portfolio, nonparametric kernel 


\section{Introduction}

Asset-liability management is a major component of insurance businesses. For life insurance companies, pension funds and annuity providers, the management of long term asset-liability depends crucially on the availability of long dated fixed income securities to cover future obligations that can span over 10-30 years. Among a variety of interest rate related securities and derivatives, long dated government bonds hold a special place in the portfolios of traditional annuity firms. Under the risk-neutral pricing framework, bond securities are priced by modelling the underlying interest rate dynamics, such as short rate models, under the risk-neutral measure. In this paper we propose to apply a more general framework, namely the benchmark approach (see Platen and Heath (2010)), to price and hedge long dated zero coupon bonds. Under the benchmark approach, the numéraire is the growth optimal portfolio (GOP), which can be approximated by a well-diversified equity index, and the pricing measure is the real-world probability measure. One of the consequences is that under realistic long term modelling the price dynamics of a long dated zero coupon bond depends not only on interest rate risk, but also on the market price of equity risk.

Short rate models have been studied intensely in the literature. Starting from the seminal work of Merton (1973) in continuous time finance, they have become fundamental and important price determinants when evaluating interest rate contingent claims and hedging interest rate risk. Modelling stochastic interest rates has also become increasingly important in solving important actuarial problems. For example, Peng et al. (2012) price guaranteed minimum withdrawal benefits in variable annuities under stochastic interest rates. In Nowak and Romaniuk (2013), stochastic interest rates are taken into account when pricing and simulating catastrophe bonds. Many continuous time models have been put forward to reflect the behaviour of interest rates. These include models introduced in Brennan and Schwartz (1977, 1979, 1980); Vasicek (1977); Dothan (1978); Cox et al. (1985); Longstaff (1989); Black et al. (1990); Hull and White (1992) and Longstaff and Schwartz (1992). For more recent accounts in this area and overviews on existing models one can refer to Stanton (1997); Rebonato (1998) and Brigo and Mercurio (2001). Despite the existence of a large number of short-term interest rate models, the model choice for applications in pricing specific fixed income securities is typically made on a case by case basis. Often different products motivate different models. By choosing a model one aims for realistic behaviour, ease of implementation, and also analytical tractability. Each product typically requires a model which combines the above features, and in some way outperforms the competing models. A more advanced model should have the property that it works well for a wide range of products and also over long time periods. Note that International Financial Reporting Standards (IFRS) and International Accounting Standards (IAS) ${ }^{1}$ recognise the importance of valuation and hedging of long dated derivatives, e.g. pensions, investments, zero coupon bonds and other fixed income securities, which appear in the market exposure of many firms. Long dated derivatives are not well covered by the currently favoured risk neutral pricing and hedging methodology, as argued in Platen and Heath (2010).

This paper aims to search for suitable models that deal well with pricing and hedging of long dated zero coupon bonds under stochastic interest rates, by employing the benchmark approach. We place ourselves in a hybrid framework, to model simultaneously both the dynamics of the short rate and the growth optimal portfolio (GOP), used as numéraire portfolio or benchmark for pricing purposes and also for hedging. For modelling interest rate risk we consider different short rate models. These include the Vasicek model, see Vasicek (1977); the CIR model, see Cox et al. (1985); the 3/2 model, introduced in Ahn and Gao (1999); the Dothan model, see Dothan (1978) and the Craddock-Lennox short rate model, see Craddock and Lennox (2007). For the GOP modelling we build upon the time dependent

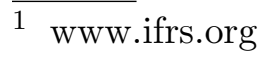


constant elasticity of variance (TCEV) model introduced in Baldeaux et al. (2014), which models the GOP and is extended in our analysis by taking into account stochastic interest rate risk. The TCEV model represents a model class which nests several known model specifications. Among other models it includes the constant elasticity of variance (CEV) model, see Cox (1996), and the minimal market model (MMM), see Platen and Heath (2010). When using real world pricing, derivative price processes, when denominated in units of the GOP, become martingales under the real world probability measure. Thus, contingent claim prices can be calculated via conditional expectations using the real world probability measure. Under classical assumptions the resulting prices coincide with classical risk neutral prices, however, real world pricing makes also perfect sense for models beyond the classical paradigm, see Du and Platen (2014). Ceci et al. (2014) suggest another application of the benchmark approach to develop locally risk-minimizing hedging strategies in a semimartingale financial market model under partial information. For more details on the GOP and the numéraire portfolio we refer to Kelly (1956), see also Latané (1959), Breiman (1960), Thorp (1961), Markowitz (1976), and Long (1990). Further studies on valuation portfolios in asset-liability management in the actuarial context can be found e.g. in Buchwalder et al. (2007) and Lim and Wong (2010).

In the current paper we consider the problem of pricing long dated contingent claims using real world pricing under stochastic interest rates and a realistic model for the numéraire portfolio, the GOP, for which a risk neutral probability measure does not exist. The latter provides a more realistic model for the long term dynamics of the financial market than is obtainable under the classical risk neutral paradigm. We use a non-parametric kernel-based technique to estimate the diffusion coefficient function, and potentially, the drift coefficient, of the short rate model, and refer to Florens-Zmirou (1993), Stanton (1997), Jiang and Knight (1997), Soulier (1998) and Jacod (2000) for details on the estimation technique. We aim to find a model that is tractable, fits well the historical data, i.e., cannot be easily falsified or rejected as other models based on the data fit. We show that the $3 / 2$ model tends to capture better the dynamics of the short-term interest rates than the competing models. We also apply the non-parametric technique to estimate the diffusion coefficient function of the discounted GOP, without assuming any particular scalar diffusion dynamics. It turns out that the diffusion coefficient function of the TCEV model fits surprisingly well the non-parametrically estimated diffusion coefficient function. ${ }^{2}$

To identify the best performing model for the purposes of long dated bond pricing we evaluate and compare historical market bond prices with theoretical prices derived by the benchmark approach. It turns out that the proposed hybrid model is highly tractable. It allows us to derive closed-form solutions for bond prices and hedge ratios. Furthermore, the hybrid model with the $3 / 2$ dynamics for the short rate leads to the smallest prices when compared to other alternative models. Finally, this model performs successfully when empirically hedging long dated zero coupon bonds by employing short-maturity zero coupon bonds, the savings account and the GOP for the hedge.

This paper is organised as follows. Section 2 develops a hybrid modelling framework for stochastic short-term interest rates together with the GOP. Section 3 discusses the non-parametric kernel-based technique, used to estimate the drift and the diffusion coefficient functions in the short rate and the GOP dynamics. Estimation results are presented in Section 4. Section 5 summarises the real world pricing approach and shows how to calculate prices of zero coupon bonds under the hybrid framework with different short rate specifications. Pricing results and performance are evaluated in Section 6 by comparing model prices to market prices. Finally, Section 7 discusses hedging results obtained for the best performing model, and Section 8 concludes the paper.

$\overline{2}$ This holds for the GOP denominated in different currencies, whereas this study focuses on USD denomination of the GOP. 


\section{Modelling Framework}

This section describes a hybrid framework for modelling the dynamics of stochastic short rates and the GOP simultaneously. For the dynamics of short rates we consider a general econometric framework. It nests several well-known model specifications, including the Vasicek model (see Vasicek (1977)), the CIR model (see Cox et al. (1985)), 3/2 model, introduced in Ahn and Gao (1999) and the CraddockLennox short rate model, see Craddock and Lennox (2007). For the GOP we use the time dependent constant elasticity of variance (TCEV) model introduced in Baldeaux et al. (2014), which assumes that the drift of the discounted GOP is an exponentially growing function of time.

\subsection{Short Rate Models}

We employ here a standard econometric framework to compare the performance of a variety of wellknown interest rate models in capturing the stochastic behaviour of the short-term interest rate. To specify the most general model for the short rate dynamics studied in this paper, we consider the following SDE:

$$
d r_{t}=g\left(r_{t}\right) d t+\sigma r_{t}^{\alpha} d W_{t}
$$

where $\sigma>0, \alpha \in[0, \infty)$ and $W=\left\{W_{t}, t \geq 0\right\}$ is a standard Wiener process on a filtered probability space $(\Omega, \mathcal{A}, \underline{\mathcal{A}}, P)$ with filtration $\underline{\mathcal{A}}=(\mathcal{A})_{t>0}$ satisfying the usual conditions, see Karatzas and Shreve (1991). These Markovian dynamics imply that the conditional mean and variance of changes in the short rate depend only on the level $r_{t}$. This allows us to concentrate on reasonably tractable short rate models. Depending on the specification of the drift $g\left(r_{t}\right)$ and the diffusion coefficient function $\sigma r_{t}^{\alpha}$, we can select a particular model. We concentrate on five models considered in the literature, with specifications listed in Table 1.

Table 1

Alternative model specifications for the short-term interest rate dynamics.

\begin{tabular}{l|l|l|l|l}
\hline \hline Model & SDE & $\alpha$ & $g\left(r_{t}\right)$ & Param. Constr. \\
\hline 1. Vasicek & $d r_{t}=\left(a-b r_{t}\right) d t+\sigma d W_{t}$ & 0 & $\left(a-b r_{t}\right)$ & $a, b, \sigma>0$ \\
2. CIR & $d r_{t}=\kappa\left(\theta-r_{t}\right) d t+\sigma \sqrt{r_{t}} d W_{t}$ & $1 / 2$ & $\kappa\left(\theta-r_{t}\right)$ & $\kappa, \theta, \sigma>0$ \\
3. 3/2 & $d r_{t}=\left(p r_{t}+q r_{t}^{2}\right) d t+\sigma r_{t}^{3 / 2} d W_{t}$ & $3 / 2$ & $\left(p r_{t}+q r_{t}^{2}\right)$ & $q<\sigma^{2}$ \\
4. Dothan & $d r_{t}=\lambda r_{t} d t+\sigma r_{t} d W_{t}$ & 1 & $\lambda r_{t}$ & $\sigma>0, \lambda \in \mathbb{R}$ \\
5. C\&L & $\mathrm{d} r_{t}=2 r_{t} \sqrt{\xi} \cot \left(\frac{r_{t}}{\sqrt{\xi}}\right) d t+\sqrt{2 \xi r_{t}} d W_{t}$ & $1 / 2$ & $2 r_{t} \sqrt{\xi} \cot \left(\frac{r_{t}}{\sqrt{\xi}}\right)$ & $r_{0}>0$ \\
\hline \hline
\end{tabular}

Model 1 is the Vasicek (1977) interest rate model introduced in Vasicek (1977). It uses the OrnsteinUhlenbeck process to provide an equilibrium model for short rates. It yields a stationary Gaussian process, which assumes that the short rate $r_{t}$ is linearly mean reverting. For $t$ going to infinity, the expected short rate tends to the long term average rate $a / b$. In the literature it is argued that the drawback of the Vasicek model is that the short rate $r_{t}$ can become negative. However, the analytical tractability, which is implied by its Gaussian transition density, is hardly achieved when assuming other dynamics for the process $r$. The Vasicek model and its generalisations have been used intensely to value bond options, futures, futures options and other types of contingent claims; see e.g. Jamshidian (1990) and Gibson and Schwartz (1990).

Model 2 is the CIR model, which uses the square root process for the short rate dynamics. It appears in Cox et al. (1985) as a single-factor general equilibrium short rate model. Here, we follow the presentation in Brigo and Mercurio (2001). This model has been a benchmark model for short-rate 
dynamics because of its analytical tractability and the fact that, contrary to the Vasicek model, the short rate is always nonnegative. It has been applied in constructing tractable valuation models for interest-rate-sensitive contingent claims, including discount bond options, futures and futures options and swaps, see e.g. Cox et al. (1985), Ramaswamy and Sundaresan (1986), and Longstaff (1990).

Model 3 is the 3/2 model introduced in Ahn and Gao (1999), see also Platen (1999), for modelling the short rate dynamics. Here, we follow the representation in Carr and Sun (2007), who employed this type of model to describe the instantaneous variance in equity models. It differs from the other popular short rate models in the power $\alpha=\frac{3}{2}>1$ of the diffusion coefficient. Such a high power in the diffusion coefficient function has been supported by an earlier empirical result in Chan et al. (1992), who show via parametric methods that models with power $\alpha \geq 1$ capture the dynamics of the short-term interest rate better than those which assume $\alpha<1$.

Model 4 is the Dothan model, introduced in Dothan (1978), where we follow the presentation in Pintoux and Privault (2011). Here, the short-term interest rate process follows a geometric Brownian motion and, therefore, uses $\alpha=1$. This model leads to the interest rate being conditionally log-normally distributed, which implies that the short rate cannot become negative and, therefore, overcomes a main criticism of the Vasicek model. However, the process is only mean reverting for $\lambda<0$ with mean-reversion level equal to zero. The resulting dynamics do not seem to be realistic for long time periods, since the variance of the log-short rate is growing proportionally to time. Nevertheless, the Dothan model appears to be analytically tractable. In fact, it seems to be the only log-normal short rate model in the literature with analytical formulas for pure discount bonds, see Brigo and Mercurio (2001) and Pintoux and Privault (2011).

Model 5, which in the following will be referred to as the $C \& L$ model, is the Craddock-Lennox short rate model. It was introduced in Craddock and Lennox (2007), who constructed short rate models using Lie symmetry group methods and showed that bond prices can be derived in closed form for this model. To our knowledge, the $C \& L$ model has not been empirically tested so far against different alternative model specifications.

\subsection{Modelling the GOP}

First, let us recall the general stochastic differential equation (SDE) for the dynamics of the GOP in a continuous financial market. We denote by $\bar{S}_{t}^{*}$ the value of the discounted GOP at time $t$. Following Platen and Heath (2010), the discounted GOP $\bar{S}_{t}^{*}$ satisfies in a continuous financial market the SDE

$$
d \bar{S}_{t}^{*}=\bar{S}_{t}^{*} \theta_{t}\left(\theta_{t} d t+d \bar{W}_{t}\right)
$$

for $t \geq 0$. Here, $\bar{S}_{0}^{*} \geq 0$ is the initial value and $\theta_{t}$ denotes the volatility of the GOP or market price of risk, where $\bar{W}=\left\{\bar{W}_{t}, t \geq 0\right\}$ denotes a standard Wiener process.

Following Baldeaux et al. (2014), we introduce the so-called time dependent constant elasticity of variance (TCEV) model, which assumes that the GOP volatility, or market price of risk, is of the form

$$
\theta_{t}=g\left(\frac{\bar{S}_{t}^{*}}{\alpha_{t}}\right)
$$

Here $g(\cdot)$ is a given function, and $\alpha_{t}$ is a deterministic function of time that is defined below. Using 
this notation, we can write the following SDE for the discounted GOP:

$$
d \bar{S}_{t}^{*}=\bar{S}_{t}^{*} g\left(\frac{\bar{S}_{t}^{*}}{\alpha_{t}}\right)^{2} d t+\bar{S}_{t}^{*} g\left(\frac{\bar{S}_{t}^{*}}{\alpha_{t}}\right) d \bar{W}_{t} .
$$

For our model we assume that $\alpha_{t}$ is a function of time growing exponentially like the average of the discounted GOP, that is,

$$
\alpha_{t}=\alpha_{0} \exp \{\eta t\}
$$

with $\eta>0$. We remark, assuming that

$$
g\left(\frac{\bar{S}_{t}^{*}}{\alpha_{t}}\right)=c\left(\frac{\bar{S}_{t}^{*}}{\alpha_{t}}\right)^{a-1}
$$

where $a \in(-\infty, 1)$ and $c>0$, leads to the TCEV model and by $(2.4)$ to the SDE

$$
d \bar{S}_{t}^{*}=c^{2} \alpha_{t}^{2-2 a}\left(\bar{S}_{t}^{*}\right)^{2 a-1} d t+c \alpha_{t}^{1-a}\left(\bar{S}_{t}^{*}\right)^{a} d \bar{W}_{t}
$$

for $t \in[0, \infty)$ and $\bar{S}_{0}^{*}>0$. For the purpose of our analysis we also introduce the normalized GOP process $Y=\left\{Y_{t}=\frac{\bar{S}_{t}^{*}}{\alpha_{t}}, t \geq 0\right\}$, which follows the SDE

$$
d Y_{t}=\left(c^{2} Y_{t}^{2 a-1}-\eta Y_{t}\right) d t+c Y_{t}^{a} d \bar{W}_{t}
$$

for $t \geq 0$ and $Y_{0}=y>0$, and exponent $a \in(-\infty, 1)$. Note that $Y=\left\{Y_{t}, t \geq 0\right\}$ is time homogenous. Using the SDE (2.8) and the Itto formula, one can easily verify that $\bar{S}_{t}^{*}$ can be represented as the product

$$
\bar{S}_{t}^{*}=\alpha_{t} Y_{t} .
$$

Note that for $a=\frac{1}{2}$ and $c=1$ the TCEV model recovers the minimal market model in Platen and Heath (2010).

\section{Nonparametric Estimation of Drift and Diffusion Coefficient Functions}

Non-parametric kernel-based estimation of diffusion coefficient functions has been introduced in FlorensZmirou (1993) and is discussed in several studies; see e.g. Soulier (1998) and Jacod (2000). Following the methodology of Stanton (1997) and Jiang and Knight (1997), we apply the nonparametric kernelbased technique to the estimation of the diffusion coefficients in the dynamics of the short rate, as well as the equity index, as discussed in Ignatieva and Platen (2012) and Baldeaux et al. (2014).

Given $n$ sample points $\left(x_{t_{1}}, \ldots, x_{t_{n}}\right)$, the kernel estimation density $\hat{f}\left(x, h_{n}\right)$ is defined as

$$
\hat{f}\left(x, h_{n}\right)=\frac{1}{n h_{n}} \sum_{i=1}^{n} K\left(\frac{x-x_{t_{i}}}{h_{n}}\right)
$$

where $K(\cdot)$ is a kernel function and $h_{n}$ is a bandwidth parameter controlling the degree of smoothness of the estimator. In this paper, we employ a Gaussian kernel function, i.e. we set $K(z)=$ $(2 \pi)^{-\frac{1}{2}} \exp \left\{-\frac{1}{2} z^{2}\right\}$. It has been shown in the literature, that the specific choice of the kernel $K(\cdot)$ 
does not affect much the performance of the resulting estimator. In fact, as argued in Epanechnikov (1969), any reasonable kernel gives almost optimal results. For the bandwidth $h_{n}$, one typically chooses the bandwidth which minimizes the asymptotic mean integrated squared error (MISE), see Wand and Jones (1995) and Botev et al. (2010). For the purpose of our analysis we use a Gaussian kernel with cross-validated bandwidth selected using Silverman (1986)'s rule of thumb, that is, $h_{n}=h_{c} \hat{\sigma} n^{-\frac{1}{5}}$, where $\hat{\sigma}$ is the dispersion of observations, $n$ is the number of sample points and $h_{c}=1.06$.

We can calculate the estimator of the drift and the squared diffusion coefficient functions, as suggested by Stanton (1997), in the following way:

$$
\begin{aligned}
& \mu_{1}\left(x_{t_{i-1}}\right)=\frac{1}{t_{i}-t_{i-1}} E\left(\left(x_{t_{i}}-x_{t_{i-1}}\right) \mid x_{t_{i-1}}=x\right)+O\left(t_{i}-t_{i-1}\right) \\
& \sigma_{1}^{2}\left(x_{t_{i-1}}\right)=\frac{1}{t_{i}-t_{i-1}} E\left(\left(x_{t_{i}}-x_{t_{i-1}}\right)^{2} \mid x_{t_{i-1}}=x\right)+O\left(t_{i}-t_{i-1}\right),
\end{aligned}
$$

using the approximations

$$
E\left(\left(x_{t_{i}}-x_{t_{i-1}}\right) \mid x_{t_{i-1}}=x\right) \approx \frac{\sum_{i=1}^{n}\left(x_{t_{i}}-x_{t_{i-1}}\right) K\left(\frac{x-x_{t_{i-1}}}{h}\right)}{\sum_{i=1}^{n} K\left(\frac{x-x_{t_{i-1}}}{h}\right)}
$$

and

$$
E\left(\left(x_{t_{i}}-x_{t_{i-1}}\right)^{2} \mid x_{t_{i-1}}=x\right) \approx \frac{\sum_{i=1}^{n}\left(x_{t_{i}}-x_{t_{i-1}}\right)^{2} K\left(\frac{x-x_{t_{i-1}}}{h}\right)}{\sum_{i=1}^{n} K\left(\frac{x-x_{t_{i-1}}}{h}\right)},
$$

respectively, where $t_{i}-t_{i-1}$ denotes the time step size between successive observations. In addition to the above first order approximations, Stanton (1997) developed higher order approximations based on Taylor expansions. However, he showed that using approximations of higher order does not affect the order of convergence but may improve the approximations for a chosen time step $t_{i}-t_{i-1}$. For the purpose of our analysis we do not require higher order approximations to fit a parametric form to the estimated functions for the drift and diffusion coefficients and, thus, the first order approximation (3.2) will be sufficient.

\section{Estimation Results}

This section discusses the data used in our empirical analysis and reports estimation results for the hybrid model. We start with the estimation of the short rate models followed by the estimation of the GOP. Both methodologies will apply the nonparametric estimation procedure described in Section 3.

\subsection{Short Rate Data}

In our empirical analysis we will use daily (3-month) T-Bill rates obtained from Datastream Thomson Financial. The observation period covers the time period from January 1973 to July 2010. Figure 1 shows the interest rate level (top left panel) and interest rate changes (top right panel), defined as $r_{t+\Delta}-r_{t}$ with $\Delta$ corresponding to one day. One observes that during the time period from 1978 to 1985 , 

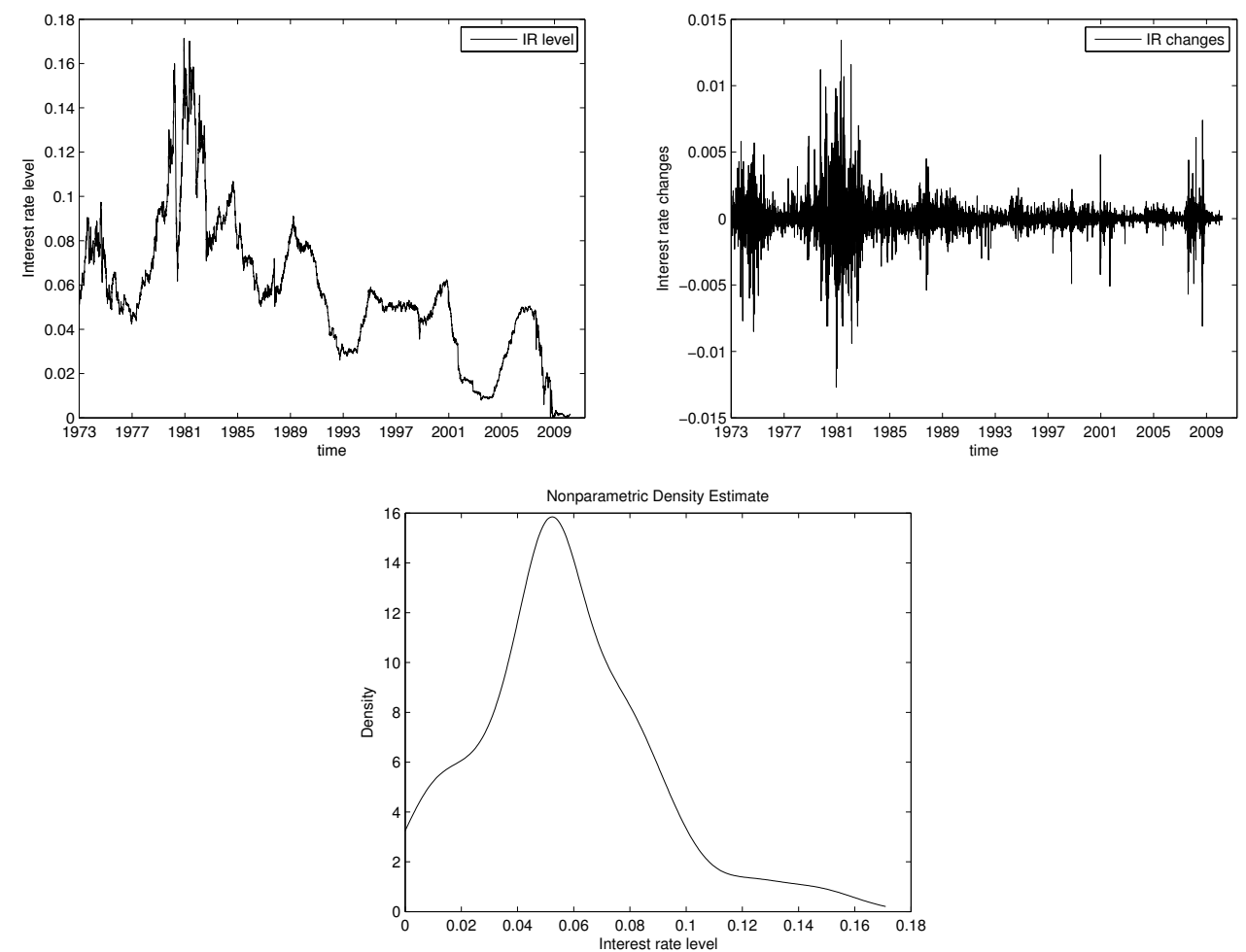

Fig. 1. Interest rate level (top left panel) and interest rate changes (top right panel); nonparametric density estimate (bottom panel).

short rates are particularly volatile. During this time the inflation drove US yields to their all-time high in 1981. While the volatility decreases gradually towards the end of the observation period, it is interesting to note that the short rates are trending downwards as well.

Table 2

Summary statistics for the short rate levels and short rate changes for the US.

\begin{tabular}{|c|c|c|c|c|c|}
\hline & Mean & Std.Dev. & Skewness & Kurtosis & First Autocorr. \\
\hline$r_{t}$ & 0.0569 & 0.0313 & 0.6811 & 3.9260 & 0.9992 \\
\hline$r_{t+\Delta}-r_{t}$ & -0.0000 & 0.0011 & 0.1797 & 27.0850 & 0.1285 \\
\hline
\end{tabular}

Table 2 provides summary statistics for the short rate levels and short rate changes. It shows that the kurtosis of daily 3 -month T-bill rate increments exceeds 27 , while the skewness is positive indicating a high level of non-normality. Furthermore, as an illustration of the nonparametric kernel methodology, and to give some idea of the distribution of interest rates, the probability density estimate for the daily changes is shown in the bottom panel of Figure 1. One observes a long right tail in the distribution of short rate increments, which together with moment statistics indicates that the distribution is leptokurtic.

\subsection{Estimating Short Rate Models}

To estimate the parameters of the drift and diffusion coefficient functions of the short rate models we apply the same methodology as for the discounted equity indices. Thereby, we estimate not only the diffusion but also the drift coefficient function since drift parameters are required for the pricing of derivative products, as will be discussed below. 

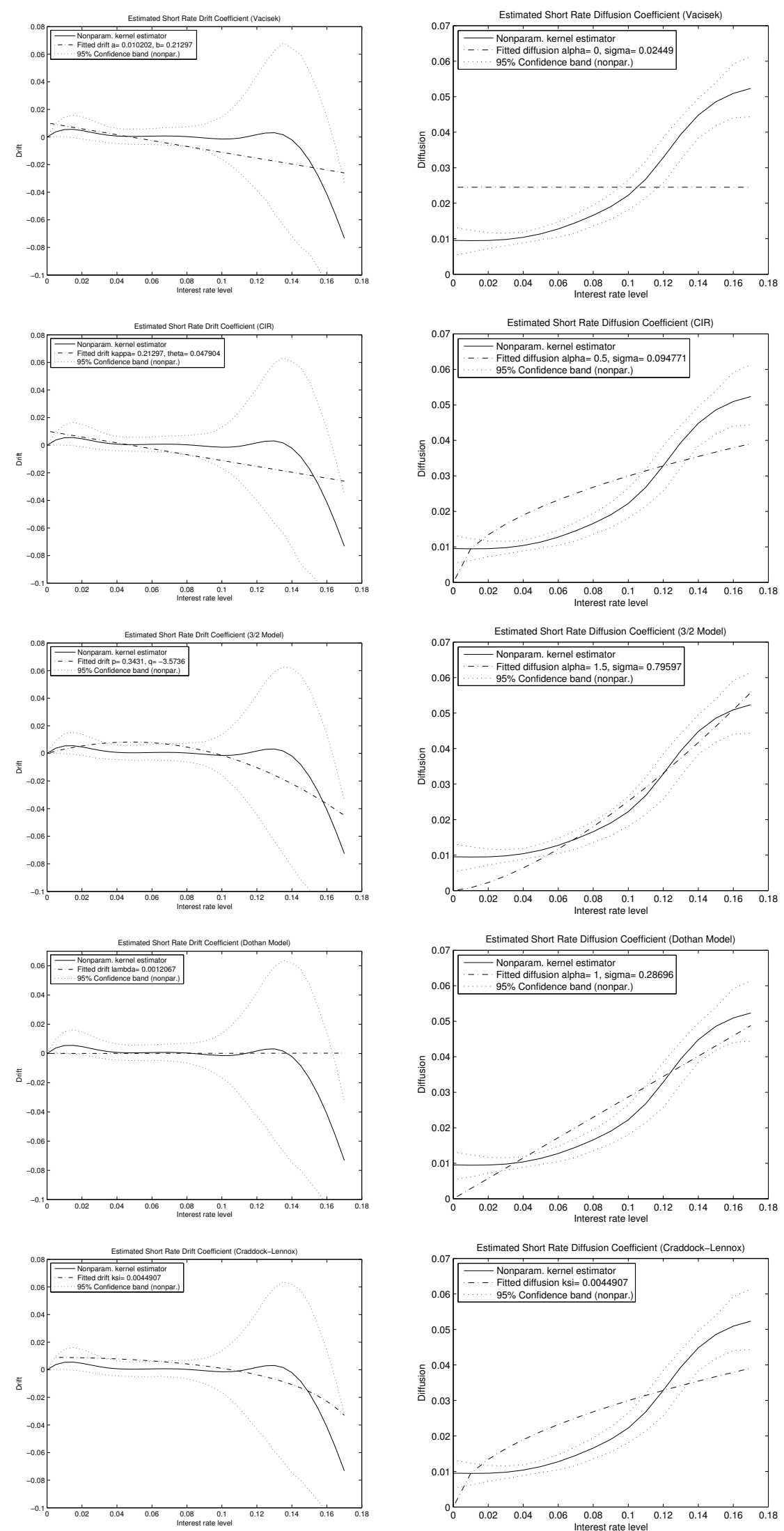

Fig. 2. Nonparametric estimates of the drift coefficient function (left panels) and the diffusion coefficient function (right panels) for the models: Vasicek, CIR, 3/2, Dothan, Craddock-Lennox (from top to bottom). 
In the first step we estimate the parameters of the diffusion coefficient function $\sigma r_{t}^{\alpha}$ in the short rate dynamics (2.1) in order to decide on the parametric model which fits the data best. The estimated coefficient for the constant $\sigma$ and the diffusion coefficient power $\alpha$ are $\sigma=0.6131$ and $\alpha=1.3694$, respectively. Thus, by just looking at the estimated power, it turns out that among all models under consideration the diffusion coefficient of the short rate can be captured most appropriately using the $3 / 2$ model, as the power of its diffusion coefficient is closest to the estimated unrestricted diffusion coefficient power.

Table 3

Nonparametric estimates for the diffusion constant and drift parameters in the interest rate dynamics.

\begin{tabular}{|c|c|c|c|c|c|c|c|c|c|c|}
\hline & \multicolumn{4}{|c|}{ Diffusion Param. } & \multicolumn{6}{|c|}{ Drift Parameters } \\
\hline & $\sigma$ & $\alpha$ & $a$ & $b$ & $\kappa$ & $\theta$ & $\lambda$ & $p$ & $q$ & $\xi=\sigma^{2} / 2$ \\
\hline Vasicek & 0.0245 & 0 & 0.0102 & 0.2130 & - & - & - & - & - & - \\
\hline CIR & 0.0938 & $1 / 2$ & - & - & 0.2130 & 0.0479 & - & - & - & - \\
\hline $3 / 2$ & 0.7960 & $3 / 2$ & - & - & - & - & - & 0.3431 & -3.5736 & - \\
\hline Dothan & 0.2870 & 1 & - & - & - & - & -0.0012 & - & - & - \\
\hline C\&L & 0.0938 & $1 / 2$ & - & - & - & - & - & - & - & 0.0045 \\
\hline
\end{tabular}

Now we assume a certain model specification. That is, we fix the diffusion coefficient power $\alpha$ to 0 , $1 / 2,1,3 / 2$ and $1 / 2$ for the Vasicek, CIR, 3/2, Dothan, and C\&L model, respectively, and estimate only the diffusion constant and the drift (restricted estimation). The results of the fitted parameter estimates obtained using non-linear least squares are summarised in Table 3. The estimated drift and diffusion coefficient functions for the Vasicek, CIR, 3/2, Dothan, and Craddock-Lennox models (from top to bottom) are plotted in the left, respectively, the right panel of Figure 2 using a solid line. ${ }^{3}$ The fitted diffusion coefficient functions for the respective model obtained, using the parameter estimates displayed in Table 3, are shown in these figures by using dashed-dotted lines.

The figure also shows a pointwise 95\% confidence band (dotted line), calculated using 1000 iterations of the block bootstrap algorithm, see Künsch (1989). In order to preserve serial dependence in the data we apply the moving block bootstrap method which uses blocks of observations rather than single observations in the bootstrap algorithm. The algorithm resamples the observed time series using approximately independent (non-overlapping) moving blocks of length $l$. For $n$ observations, we consider $k$ blocks of length $l(n=l k)$, constructed in the following way: Block one comprises observations from 1 to $l$, block two comprises observations from $l+1$ to $2 l$, etc. The last block $k$ comprises observations from $n-l+1$ to $n$. The estimation algorithms draws $k$ blocks with replacement from a set of blocks. Aligning these blocks in the order they were picked provides us with the bootstrap observations, which can then be used to compute standard errors and confidence bands.

Comparing visually the results for the parameter estimates with the diffusion coefficient function plots, we observe that the fitted diffusion coefficient function line for the $3 / 2$ model falls almost entirely into the estimated $95 \%$ confidence band and thereby, seems to outperform its competitors. It indicates that the $3 / 2$ model is likely to be a good candidate for describing the short rate dynamics when aiming for a simple scalar diffusion short rate model. This makes the short rate model proposed in Ahn and Gao (1999), as well as Platen (1999), reasonably realistic.

$\overline{3}$ From the figure we observe that the estimated diffusion coefficient is greater than zero for $r_{t}=0$, which suggests that no constraint (that the diffusion coefficient is zero for $r_{t}=0$ ) is required for the estimation of the diffusion coefficient function. However, in order to prevent interest rates from becoming negative, we impose this constraint, as suggested in Stanton (1997) for the estimation of the drift coefficient function. 

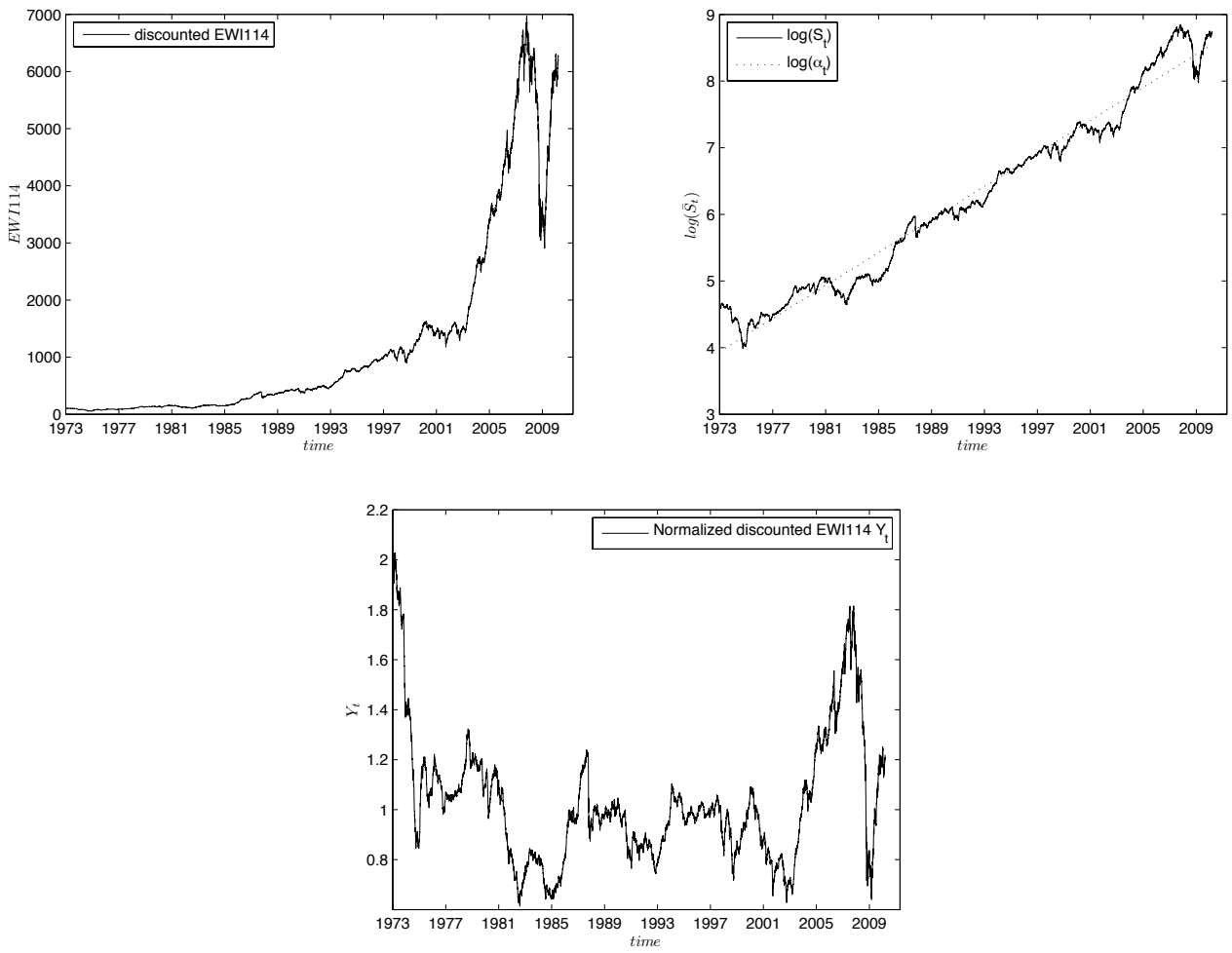

Fig. 3. Upper left panel: Discounted EWI114 $\bar{S}_{t}^{*}$ as function of time. Upper right panel: Logarithm of the discounted EWI114, $\ln \left(\bar{S}_{t}^{*}\right)$, which is on average a linearly growing function of time. Lower panel: Normalised discounted EWI114 $Y_{t}=\bar{S}_{t}^{*} / \alpha_{t}$.
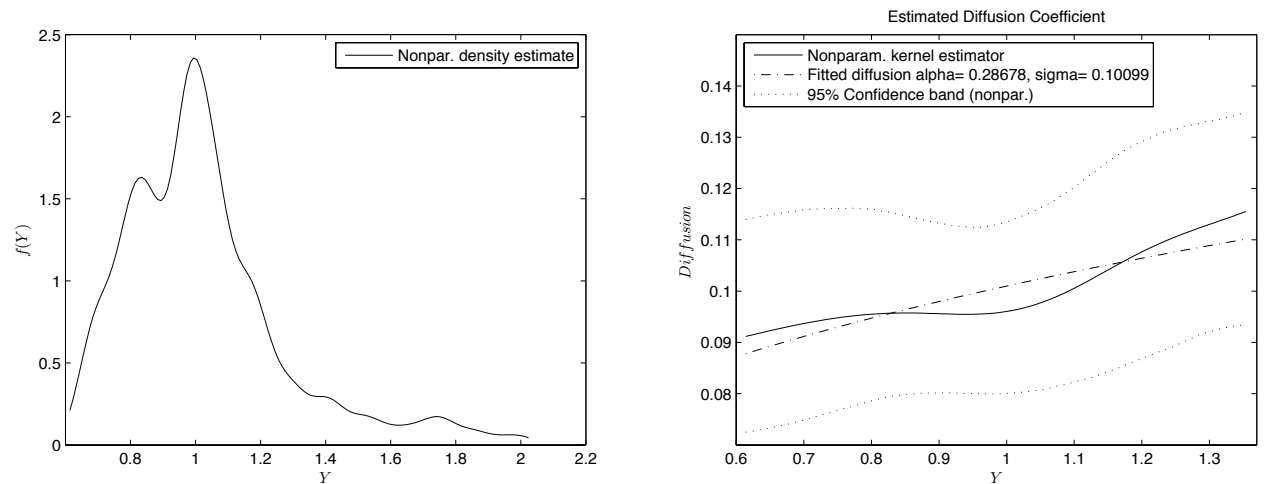

Fig. 4. Nonparametric density estimate (left panel) and diffusion coefficient estimate (right panel) for the normalised discounted EWI114 denominated in USD.

\subsection{Estimating the GOP}

As equity index and approximation of the GOP, we consider the well diversified equi-weighted index, the EWI114, which is available on a daily basis for the time period from January 1973 to July 2010. In its construction in Platen and Rendek (2012) the EWI114 uses industry sub-sector indices as constituents provided by Datastream Thomson Financial. Here we discuss estimation results for the GOP. 


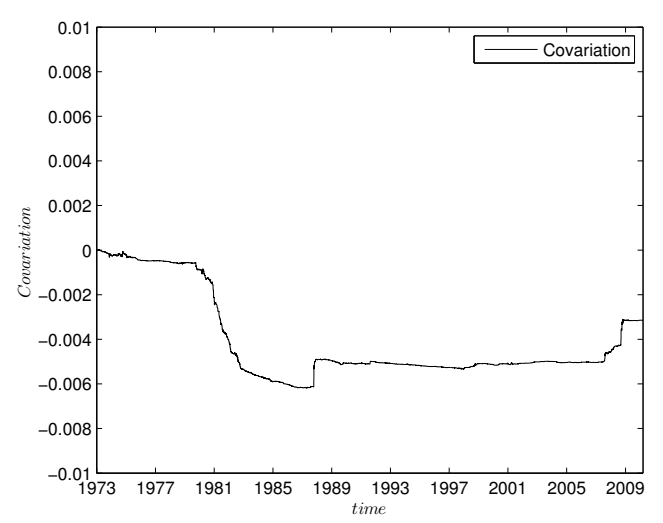

Fig. 5. Covariation between the logarithms of the EWI114 and the US short rate.

The discounted EWI114, denominated in USD, is plotted in the upper left panel of Figure $3 .{ }^{4}$ In order to obtain an exponentially increasing drift function $\alpha_{t}$, which is given by (2.5), we have to estimate the scaling parameter $\alpha_{0}$ and the net growth rate $\eta$. For this purpose we apply a least squares estimation to fit the $\operatorname{logarithm} \ln \left(\alpha_{t}\right)$ to the logarithm $\ln \left(S_{t}^{*}\right)$, as shown in the upper right panel of Figure 3 . The resulting parameter values are $\alpha_{0}=51.3455$ and $\eta=0.1239$. Given the function $\alpha_{t}$, the normalised discounted GOP can be computed as $Y_{t}=\bar{S}_{t}^{*} / \alpha_{t}$. We plot this normalised process in the lower panel of Figure 3. $Y_{t}$ can now be used to estimate the diffusion coefficient function in a non-parametric way, as described in Section 3. The kernel density is estimated using a Gaussian kernel with a 'rule-of-thumb' bandwidth and is plotted in the left panel of Figure 4. The resulting estimated approximation of the diffusion coefficient functions is plotted in the right panel of Figure 4. Fitting the estimated diffusion coefficient function to the functional form assumed for the diffusion coefficient function $\left(c Y^{a}\right)$ of the process $Y$, we obtain for the parameter estimates of $c$ and $a$ the values 0.1010 and 0.2868 , respectively. The fitted diffusion coefficient function is represented by the dashed-dotted line in the right panel of Figure 4. Again, the dotted lines represent a pointwise 95\% confidence band, calculated using 1000 iterations of the block bootstrap algorithm.

Finally, comparing the results for the parameter estimates with the diffusion coefficient function plot, we observe that the fitted diffusion line (dashed-dotted line), obtained by using parameter estimates of $c=0.1010$ and $a=0.2868$, falls into the estimated $95 \%$ confidence band for the TCEV diffusion process, indicating that the TCEV model is likely to be a good candidate model for describing the normalised EWI114 dynamics.

\section{$5 \quad$ Zero Coupon Bond Pricing}

We use $B_{t}$ to denote the value of the savings account at time $t$, assuming

$$
d B_{t}=r_{t} B_{t} d t, t \geq 0
$$

and $B_{0}=1$. The adapted short rate process is denoted by $r=\left\{r_{t}, t \geq 0\right\}$. In Figure 5 we plot the covariation between the logarithm of the discounted EWI114 and the US short rate. Notice that the

\footnotetext{
$\overline{4}$ Note, in the following we apply the estimation procedure described above to the EWI114 denominated in USD. When considering denominations of the EWI114 in other currencies, as suggested in e.g. Ignatieva and Platen (2012), the results appear to be qualitatively similar.
} 
absolute values taken by the covariation process are extremely small without exhibiting any clear trend. It does not seem that the noise processes driving the short rate and the discounted equity index are significantly correlated. Therefore, for simplicity, we assume that the driving noise source of the EWI114 and that of the short rate are independent, which leads to the following assumption:

Assumption 5.1 We assume that the Wiener processes $W$ and $\bar{W}$ are independent.

This makes the market price for short rate risk zero. Of course, the case when the market price of short rate risk is not zero can be theoretically and numerically handled as well.

\subsection{Real World Pricing}

To perform pricing under the above discussed model, we are not covered by the classical risk neutral paradigm since an equivalent risk neutral probability measure does not exist for our specification of the TCEV model. More precisely, the Radon-Nikodym derivative of the putative risk neutral measure $\Lambda_{t}=\frac{\bar{S}_{0}^{*}}{S_{t}^{*}}$ can be shown to be a strict local martingale, and is, thus, not a martingale, see Platen and Heath (2010). Therefore, we briefly recall the basics of the benchmark approach; see Platen and Heath (2010), which allows pricing and hedging also in cases when the risk neutral approach fails. Here, $S_{t}^{*}=B_{t} \bar{S}_{t}^{*}$ denotes the GOP, approximated by the EWI114, at time $t$, when denominated in units of the domestic currency (here USD).

Definition 5.1 A price process $U=\left\{U_{t}, t \geq 0\right\}$ with $E\left(\frac{\left|U_{t}\right|}{S_{t}^{*}}\right)<\infty$ for $t \geq 0$, is called fair, if the corresponding benchmarked price process $\hat{U}=\left\{\hat{U}_{t}=\frac{U_{t}}{S_{t}^{*}}, t \geq 0\right\}$ forms an $(\underline{\mathcal{A}}, P)$-martingale.

Definition 5.2 We define a contingent claim $H_{T}$ that matures at a stopping time $T$ as an $\mathcal{A}_{T}-$ measurable, non-negative payoff with $E\left(\frac{H_{T}}{S_{T}^{*}} \mid \mathcal{A}_{t}\right)<\infty$ for all $t \in[0, T]$.

Following the benchmark approach, see Platen and Heath (2010), the minimal price process $U^{H_{T}}=$ $\left\{U_{t}^{H_{T}}, t \in[0, T]\right\}$ that possibly replicates a hedgeable contingent claim $H_{T}$, is given by the conditional expectation

$$
U_{t}^{H_{T}}=E\left(\frac{S_{t}^{*}}{S_{T}^{*}} H_{T} \mid \mathcal{A}_{t}\right)
$$

for $t \in[0, T]$, which represents the real world pricing formula. The process

$$
\hat{U}^{H_{T}}=\left\{\hat{U}_{t}^{H_{T}}=\frac{U_{t}^{H_{T}}}{S_{t}^{*}}, t \in[0, T]\right\}
$$

is an $(\mathcal{A}, P)$-martingale and, thus, by Definition $5.1 U^{H_{T}}$ constitutes a fair price process. In the case of not fully replicable claims Du and Platen (2014) obtain also the real world pricing formula employing the concept of benchmarked risk minimization, where benchmarked profit and losses are orthogonal to benchmarked traded wealth.

For calculating prices of derivatives, recall that for the TCEV model we have the SDE (2.7) for the discounted GOP and

$$
\bar{S}_{t}^{*}=Z_{\varphi(t)}^{\frac{1}{2-2 a}}
$$


where $Z=\left\{Z_{\varphi}, \varphi \geq 0\right\}$ is a squared Bessel process of dimension $\nu=\frac{3-2 a}{1-a}$ and the function $\varphi(t)=$ $\frac{(1-a) \alpha_{0}^{2-2 a} c^{2}}{2 \eta}(\exp \{2(1-a) \eta t\}-1)$. Furthermore, by setting $\Delta \varphi(t)=\varphi(T)-\varphi(t)$, we define

$$
\lambda\left(t, \bar{S}_{t}^{*}\right)=\frac{\left(\bar{S}_{t}^{*}\right)^{2(1-a)}}{\Delta \varphi(t)} \text { and } x(t)=\left(\frac{K}{B_{T}}\right)^{2(1-a)} \frac{1}{\Delta \varphi(t)}
$$

and note that in distribution we have

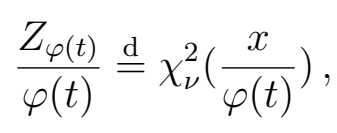

where $\chi_{\nu}^{2}(\mu)$ denotes a non-central chi-square distributed random variable with $\nu$ degrees of freedom and non-centrality parameter $\mu$.

We now present generic formulas for prices of zero coupon bonds under the short rate models discussed in this paper.

\subsection{Zero Coupon Bond Prices}

By Assumption 5.1, the price of a fair zero coupon bond is given by

$$
P(t, T)=E\left(\frac{S_{t}^{*}}{S_{T}^{*}} \mid \mathcal{A}_{t}\right)=E\left(\frac{\bar{S}_{t}^{*}}{\bar{S}_{T}^{*}} \frac{B_{t}}{B_{T}} \mid \mathcal{A}_{t}\right)=M\left(t, \bar{S}_{t}^{*}, T\right) G\left(t, r_{t}, T\right)
$$

where

$$
M\left(t, \bar{S}_{t}^{*}, T\right)=E\left(\frac{\bar{S}_{t}^{*}}{\bar{S}_{T}^{*}} \mid \mathcal{A}_{t}\right)=\Psi\left(\lambda\left(t, \bar{S}_{t}^{*}\right), \frac{1}{1-a}, 0\right),
$$

see Miller and Platen (2005), and

$$
G\left(t, r_{t}, T\right)=E\left(\frac{B_{t}}{B_{T}} \mid \mathcal{A}_{t}\right)
$$

Recall that $\Psi(\cdot, \nu, \mu)$ denotes the cumulative distribution function of a non-central chi-square distributed random variable with $\nu$ degrees of freedom and non-centrality parameter $\mu$. In the following, $G\left(t, r_{t}, T\right)$ will be referred to as the short rate contribution (SRC), whereas $M\left(t, \bar{S}_{t}^{*}, T\right)$ defines the market price of risk contribution (MPRC).

\subsection{Tractable Short Rate Models}

In this section we obtain zero coupon bond pricing formulas for the Vasicek, CIR, 3/2, Dothan and Craddock-Lennox short rate models. 


\subsubsection{Vasicek Model}

In the Vasicek model, the short rate is given by the SDE

$$
d r_{t}=\left(a-b r_{t}\right) d t+\sigma d W_{t},
$$

where $a, b$ and $\sigma$ are assumed to be positive. The following set of formulas is derived in Musiela and Rutkowski (2005). These employ the Laplace transform of $\int_{t}^{T} r_{s} d s$, which gives the formula for the zero coupon bond.

Lemma 5.1 Conditional on $\mathcal{A}_{t}$, the integral $\int_{t}^{T} r_{s} d s$ is a Gaussian random variable with mean

$$
\mu(t, T)=\frac{a}{b}(T-t)+\left(r_{t}-\frac{a}{b}\right)\left(\frac{1-\exp \{-b(T-t)\}}{b}\right)
$$

and variance

$$
\begin{aligned}
\sigma^{2}(t, T)= & -\frac{\sigma^{2}}{2 b^{3}}(1-\exp \{-b(T-t)\})^{2} \\
& +\frac{\sigma^{2}}{b^{2}}\left(T-t-\frac{1-\exp \{-b(T-t)\}}{b}\right),
\end{aligned}
$$

i.e. we have

$$
E\left(\exp \left\{-u \int_{t}^{T} r_{s} d s\right\} \mid \mathcal{A}_{t}\right)=\exp \left\{-u \mu(t, T)+\frac{u^{2}}{2} \sigma^{2}(t, T)\right\}
$$

Setting $u=1$, gives the respective formula for the $\operatorname{SRC~} G\left(t, r_{t}, T\right)$.

\subsubsection{CIR Model}

The CIR model was introduced in Cox et al. (1985), and we use here a bond price derivation given in Brigo and Mercurio (2001). Under the real world probability measure $P$ one has the SDE

$$
d r_{t}=\kappa\left(\theta-r_{t}\right) d t+\sigma \sqrt{r_{t}} d W_{t}^{1}
$$

where $r_{0}>0$ and $\kappa, \theta, \sigma$ are positive parameters.

The Laplace transform of $\int_{t}^{T} r_{s} d s$, conditional on $\mathcal{A}_{t}$, is given by the following formula, see Jeanblanc et al. (2009), Corollary 6.3.4.2.

Lemma 5.2 The Laplace transform of $\int_{t}^{T} r_{s} d s$ satisfies the formula

$$
\begin{aligned}
& E\left\{\exp \left\{-u \int_{t}^{T} r_{s} d s\right\} \mid \mathcal{A}_{t}\right\} \\
& =\exp \left\{\kappa^{2} \theta \tau / \sigma^{2}\right\}\left(\cosh \left(\frac{\gamma \tau}{2}\right)+\frac{\kappa}{\gamma} \sinh \left(\frac{\gamma \tau}{2}\right)\right)^{-2 \kappa \theta / \sigma^{2}}
\end{aligned}
$$




$$
\times \exp \left(\frac{-2 u r_{t}}{\kappa+\gamma \operatorname{coth}\left(\frac{\gamma \tau}{2}\right)}\right)
$$

where $\tau=T-t$ and $\gamma^{2}=\kappa^{2}+2 u \sigma^{2}$.

To obtain the corresponding SRC $G\left(t, r_{t}, T\right)$, we simply set $u=1$.

\subsubsection{3/2 Model}

The 3/2 model was introduced in Ahn and Gao (1999) and Platen (1999). We set

$$
d r_{t}=\left(p r_{t}+q r_{t}^{2}\right) d t+\sigma r_{t}^{3 / 2} d W_{t}
$$

where $q<\frac{\sigma^{2}}{2}$ and $\sigma>0$ to avoid explosions in $r_{t}$ : Setting $R_{t}=\frac{1}{r_{t}}$, we obtain the SDE

$$
d R_{t}=\left(\sigma^{2}-q-p R_{t}\right) d t-\sigma \sqrt{R_{t}} d W_{t}
$$

which shows that the $3 / 2$ model is the inverse of a square-root process. We now use Theorem 3 in Carr and Sun (2007) to obtain the Laplace transform of the integrated short rate process.

Lemma 5.3 The Laplace transform of $\int_{t}^{T} r_{s} d s$ has the form

$$
E\left\{\exp \left\{-u \int_{t}^{T} r_{s} d_{s}\right\} \mid \mathcal{A}_{t}\right\}=\frac{\Gamma(\gamma-\alpha)}{\Gamma(\gamma)}\left(\frac{2}{\sigma^{2} r y(t, p)}\right)^{\alpha} M\left(\alpha, \gamma, \frac{-2}{\sigma^{2} r y(t, p)}\right)
$$

where

$$
y(t, p)=\int_{t}^{T} e^{\left(t^{\prime}-t\right) p} d t^{\prime}
$$

$M(\alpha, \gamma, z)$ is the confluent hypergeometric function, see Abramowitz and Stegun (1972), with

$$
\alpha=-\left(\frac{1}{2}-\frac{q}{\sigma^{2}}\right)+\sqrt{\left(\frac{1}{2}-\frac{q}{\sigma^{2}}\right)^{2}+\frac{2 u}{\sigma^{2}}}
$$

and

$$
\gamma=2\left(\alpha+1-\frac{q}{\sigma^{2}}\right)
$$

Setting above $u=1$ yields the $\operatorname{SRC} G\left(t, r_{t}, T\right)$.

\subsubsection{Dothan Model}

The Dothan model was introduced in Dothan (1978), and we follow in this paper the presentation in Pintoux and Privault (2011). The short rate is modelled under the real world probability measure $P$ as a geometric Brownian motion via the SDE

$$
d r_{t}=\lambda r_{t} d t+\sigma r_{t} d W_{t}
$$

where $\sigma>0$ and $\lambda \in \mathbb{R}$. Zero coupon bond prices were derived in Pintoux and Privault (2011). We follow their probabilistic approach and compute the Laplace transform of the random variable $\int_{t}^{T} r_{s} d s$. 
Corollary 5.1 The Laplace transform can be given for all $p \in \mathbb{R}$, where $u>0$, by the formula

$$
\begin{aligned}
& E\left(\exp \left\{-u \int_{t}^{T} r_{s} d s\right\} \mid \mathcal{A}_{t}\right)=\frac{8 \sqrt{r_{t} u}}{\sigma^{2} \sqrt{\pi^{3} \tau}} \exp \left(-\sigma^{2} p^{2} \tau / 8+2 \pi^{2} /\left(\sigma^{2} \tau\right)\right) \\
\times & \int_{0}^{\infty} \int_{0}^{\infty} e^{-2 \xi^{2} /\left(\sigma^{2} \tau\right)} \frac{\sinh (\xi) \sin \left(4 \pi \xi /\left(\sigma^{2} \tau\right)\right)}{\sqrt{(z+\xi)\left(z+\xi^{-1}\right)}} K_{1}\left(\sqrt{8 r_{t} u} \sqrt{(z+\xi)\left(z+\xi^{-1}\right)} / \sigma\right) d \xi \frac{d z}{z^{p}}
\end{aligned}
$$

where $\tau=T-t$.

Proof. The proof can be given in the same way as Corollary 2.2 in Pintoux and Privault (2011).

The following result, which involves only a single integration, only holds for $p<1$ :

Corollary 5.2 The Laplace transform is given for all $p<1$, where $u>0$, by the expression

$$
\begin{aligned}
& E\left(\exp \left\{-u \int_{t}^{T} r_{s} d s\right\} \mid \mathcal{A}_{t}\right) \\
& =2 e^{-\sigma^{2} p^{2} \tau / 8} \int_{0}^{\infty}\left(v^{2}+8 u r_{t} / \sigma^{2}\right)^{p / 2} \theta\left(v, \frac{\sigma^{2} \tau}{4}\right) K_{p}\left(\sqrt{v^{2}+8 u r_{t} / \sigma^{2}}\right) \frac{d v}{v^{p+1}}
\end{aligned}
$$

Proof. The proof can be given in the same way as the one for Corollary 2.3 in Pintoux and Privault (2011).

Also here we obtain the respective SRC by setting $u=1$.

\subsubsection{Craddock-Lennox Model}

In Craddock and Lennox (2007), the following short rate model was analysed using Lie symmetry groups methods. The respective short rate satisfies the SDE

$$
\mathrm{d} r_{t}=2 r_{t} \sqrt{\xi} \cot \left(\frac{r_{t}}{\sqrt{\xi}}\right) d t+\sqrt{2 \xi r_{t}} d W_{t}^{1},
$$

where $r_{0} \geq 0$. The following function, the fundamental solution to the PDE for the transition density can be used in bond pricing:

$$
p_{\mu}(t, x, y)=\exp \left\{\frac{-(x+y)}{\xi t}\right\}\left(\frac{\sqrt{x}}{\sqrt{y} \xi t} I_{1}\left(\frac{2 \sqrt{x y}}{\xi t}\right)+\delta(y)\right) \frac{\sin \left(y \sqrt{\frac{\mu}{\xi}}\right)}{\sin \left(x \sqrt{\frac{\mu}{\xi}}\right)}
$$

Here, $I_{1}(\cdot)$ denotes the modified Bessel function of the third kind of order 1 and $\delta(\cdot)$ the Dirac delta measure. For the required Laplace transform Craddock and Lennox (2007) provide the following result:

Lemma 5.4 The Laplace transform of $\int_{t}^{T} r_{s} d s$ satisfies for $u>0$ the formula 


$$
E\left\{\exp \left(-u \int_{t}^{T} r_{s} d s\right) \mid \mathcal{A}_{t}\right\}=\exp \left\{-\frac{r_{t}(T-t) u}{1+\xi(T-t)^{2} u}\right\} \frac{\sin \left(\frac{\sqrt{u} r_{t}}{\sqrt{\xi}+\xi^{3 / 2}(T-t)^{2} u}\right)}{\sin \left(\frac{r_{t} \sqrt{u}}{\sqrt{\xi}}\right)}
$$

By setting $u=1$, we obtain the formula for the SRC.

\section{$6 \quad$ Pricing Results}

This section demonstrates how zero coupon bonds can be priced using the different models under consideration. Thereby, we evaluate model performance by comparing model prices and yields for bonds of different maturities to those observed in the market. In line with the concept of benchmarked risk minimization of $\mathrm{Du}$ and Platen (2014), we will identify the best performing model as the model that leads to the lowest prices compared to the market and shows benchmarked hedge errors that are orthogonal to benchmarked primary security accounts. The latter means that the benchmarked profit and loss and their products with benchmarked savings accounts are local martingales and do not exhibit systematic trends.

We illustrate the pricing of zero coupon bonds of different maturities corresponding to the tenors of 6 month, 1 year, 2 years, 5 years, 10 years, 20 years and 30 years. Thereby, prices are computed using the different model specifications, as described in Section 5.3. As an illustration, we compare model prices to market prices on four different dates that all fall into our estimation period. Market prices are provided on a monthly basis by Bloomberg for the time period from May 1991 until July 2010.

To obtain a visual impression on how model prices evolve, compared to market prices, we first consider bond prices and yields ${ }^{5}$ on several dates, followed by presenting the summary statistics for the entire sample. To visualise pricing performance, we consider bond prices and bond yields computed for five randomly selected dates in our sample period in the left, respectively, the right panel of Figure $6 .{ }^{6}$ Market prices and yields are presented using the most solid line. Visually, one observes that all models fit reasonably well the market data. However, the $3 / 2$ model tends to outperform for many periods the competing models by leading to the smallest price and highest yields. The Dothan model seems to perform worst for most of the sample periods.

In addition, Figure 7 shows in the top left panel the market price of risk contribution (MPRC) $M\left(t, \bar{S}_{t}^{*}\right)$, which enters the pricing formula (5.1) as a function of time to maturity and power $a$ in the dynamics of the GOP. The top right panel of Figure 7 shows $M\left(t, \bar{S}_{t}^{*}\right)$ as a function of time to maturity for the estimated diffusion coefficient power $a=0.2868$, which we obtained in Section 4 when estimating parameters for the GOP dynamics. The bottom left panel shows the MPRC $M\left(t, \bar{S}_{t}^{*}\right)$ as a function of the diffusion power $a$ for a fixed time to maturity of $T=15$ years. We observe that for the estimated parameter set the MPRC remains nearly constant for maturities $T$ up to 12 years (top right panel). Beyond this period, the market price of risk contribution starts to decrease markedly in value. Therefore, the MPRC does not impact the zero coupon bond price until medium maturities of the yield curve. This property of the fair zero coupon bond price results from the fact that the discounted GOP is a strict local martingale; see Platen and Heath (2010) and Platen and Bruti-Liberati (2010).

5 The yield to maturity $R(t, T)$ is defined as $R(t, T)=-\frac{\log P(t, T)}{T-t}$, where $P(t, T)$ denotes the price at time $t$ of a zero coupon bond maturing at time $T$.

${ }^{6}$ Figures covering other months from the sample period are available upon request from the authors. 

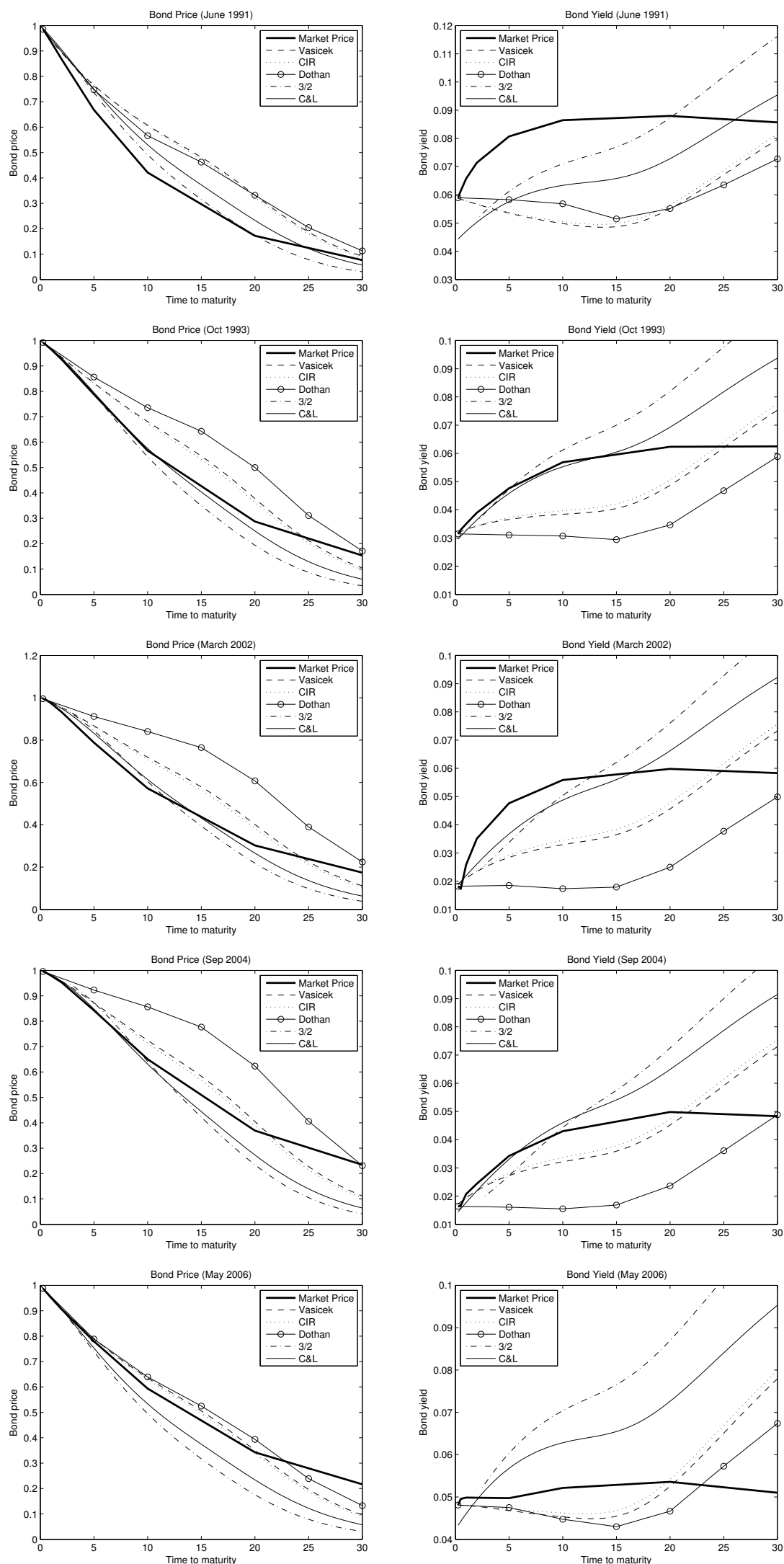

Fig. 6. Zero coupon bond prices (left panel) and yields (right panel) for bonds of different maturities and five different dates. Prices are computed under different model specifications (Vasicek, CIR, 3/2, Dothan, C\&L). Market prices are presented using solid lines. 

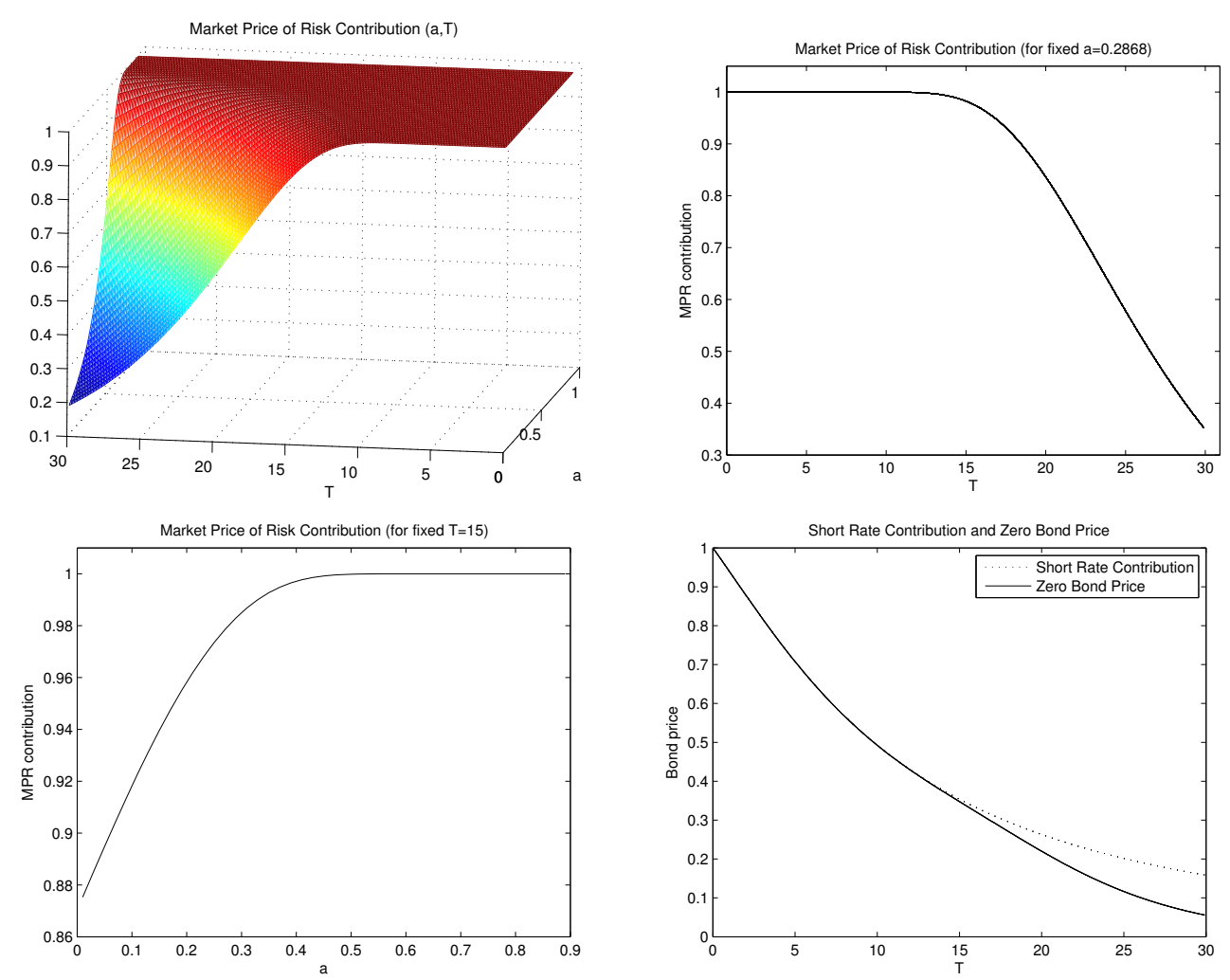

Fig. 7. Upper left panel: market price of risk contribution to the zero coupon bond price as a function of time to maturity $T$ and power $a$ in the GOP dynamics. Upper right panel: market price of risk contribution to the zero coupon bond price for estimated $a=0.2868$ and different times to maturity. Bottom left panel: market price of risk contribution to the zero coupon bond price for constant time to maturity $T=15$ years and different powers $a$. Bottom right panel: bond price and short rate contribution versus time to maturity.

For the fixed maturity of $T=15$ years the MPRC reaches a value of about one for $a$ greater than 0.4 , which again indicates that the MPRC does not impact the zero coupon bond price as soon as $a$ exceeds a particular threshold.

By using the estimated parameters and observed short rate and index value one can for all observation times (daily) calculate the respective model bond prices. To compare the overall performance of the competing models, we compute the relative pricing error $\delta_{\tau}$ for all models under consideration. It is defined as the difference between the model price and the market price, normalised by the market price, that is,

$$
\delta_{\tau}=\frac{P(t, T)^{i}-P(t, T)^{m}}{P(t, T)^{m}} .
$$

Here $P(t, T)^{i}$ defines the model price $i, i \in\{$ Vasicek,CIR,3/2,Dothan,C\&L $\} . P(t, T)^{m}$ is the market price and $\tau$ is the time to maturity, which we set equal to 6 month, 1 year, 5 years, 10 years, 20 years, 30 years for zero coupon bonds. Note that we are not comparing the absolute value $\delta_{\tau}$ since we are interested in the direction of the deviation and, thereby, aim to identify the model with the least expensive bonds compared to other models and the market. Since we use daily data we have a reasonably large data set.

To compare the performance of the models with respect to the direction of the deviation from the market price, we consider the summary statistics (mean, median, standard deviation, 5\% and 95\% quantiles) for the pricing error $\delta_{\tau}$ defined in equation (6.1). The results are summarised in Table 4, 
and Figure 9 shows the corresponding box-plots. Here, the negative (respectively positive) sign of the pricing error indicates that the model undercuts (respectively overshoots) the market price. For short maturities of 6 month, 1 year and 5 years one observes that the average pricing errors are close to zero for all considered models. However, the $3 / 2$ and the C\&L models lead to negative pricing errors indicating that these models undercut market prices as well as produce lower prices than the other competing models. When increasing the time to maturity to 10 years one observes that the $3 / 2$ model outperforms the C\&L model based on both, mean and median statistic, and again, both models lead to prices lower than the market price and lower than those of the other competing models. For the longest time to maturity of 30 years all models, except the Dothan model, exhibit negative pricing errors indicating that the market is likely to be overpriced. One notes in Table 4 that the standard deviations of the pricing errors are for the tenors up to 5 years for all models rather similar. Remarkably, for the extreme maturities the $3 / 2$ model has a significantly smaller standard deviation. The $3 / 2$ model leads to the lowest bond price (followed by the C\&L model), and thus, outperforms all competing models for long and extreme maturities.

\section{Hedging the Zero Coupon Bond}

In this section, we discuss the hedging of the zero coupon bonds. We firstly present martingale representations of the benchmarked zero coupon bond price. Using such representation, we discuss a hedging strategy involving a short maturity zero coupon bond, the savings account and the GOP. Finally, since we have identified the $3 / 2$ model as the best performing model in the sense that it leads to the lowest bond prices, we present hedging results obtained by using the respective hybrid model with the $3 / 2$ dynamics for the interest rates and the TCEV dynamics for the discounted index.

\subsection{Hedge Ratios for the 3/2 Model}

In this section we demonstrate how a zero coupon bond can be hedged by means of dynamic trading in the short maturity bond, the savings account and the GOP. We restrict ourselves to the hybrid model with $3 / 2$ dynamics for interest rates. To derive hedge ratios, we use the following notation:

$$
\begin{aligned}
V\left(r_{t}, t, T\right) & :=E\left(\exp \left\{-\int_{t}^{T} r_{s} d s\right\} \mid \mathcal{A}_{t}\right) \\
& =\frac{\Gamma(\gamma-\alpha)}{\Gamma(\gamma)}\left(\frac{2}{\sigma^{2} r y(t, p)}\right)^{\alpha} M\left(\alpha, \gamma,-\frac{2}{\sigma^{2} r y(t, p)}\right),
\end{aligned}
$$

see Lemma 5.3,

$$
u\left(t, \hat{B}_{t}, T\right)=E\left(\frac{1}{\bar{S}_{T}^{*}} \mid \mathcal{A}_{t}\right)=E\left(\hat{B}_{T} \mid \mathcal{A}_{t}\right)=\left(\bar{S}_{t}^{*}\right)^{-1} \Psi\left(\lambda\left(t, \bar{S}_{t}^{*}\right), \frac{1}{1-a}, 0\right),
$$

see (5.2), where $\hat{B}_{t}=\frac{B_{t}}{S_{t}^{*}}$. We remark that explicit formulae for $V$ and $u$ were derived in Ahn and Gao (1999) and Baldeaux et al. (2014), respectively. The following lemma produces a martingale 
representation for the benchmarked zero coupon bond

$$
\hat{P}(t, T)=\frac{P(t, T)}{S_{t}^{*}}=E\left(\frac{1}{B_{T} \bar{S}_{T}^{*}} \mid \mathcal{A}_{t}\right)=E\left(\hat{B}_{T} \mid \mathcal{A}_{t}\right) E\left(\frac{1}{B_{T}} \mid \mathcal{A}_{t}\right)=\frac{u\left(t, \hat{B}_{t}, T\right)}{B_{t}} V\left(r_{t}, t, T\right) .
$$

Corollary 7.1 Let $r=\left\{r_{t}, t \geq 0\right\}$ be given by

$$
d r_{t}=\left(p r_{t}+q r_{t}^{2}\right) d t+\sigma r_{t}^{3 / 2} d W_{t}
$$

and

$$
d \bar{S}_{t}^{*}=c^{2} \alpha_{t}^{2-2 a}\left(\bar{S}_{t}^{*}\right)^{2 a-1} d t+c \alpha_{t}^{1-a}\left(\bar{S}_{t}^{*}\right)^{a} d \bar{W}_{t}
$$

then

$$
d(\hat{P}(t, T))=\frac{1}{B_{t}}\left(\frac{\partial V\left(r_{t}, t, T\right)}{\partial r_{t}} \sigma r_{t}^{3 / 2} u\left(t, \hat{B}_{t}, T\right) d W_{t}+V\left(r_{t}, t, T\right) \frac{\partial u\left(t, \hat{B}_{t}, T\right)}{\partial \hat{B}_{t}}\left(-c \alpha_{t}^{1-a}\left(\bar{S}_{t}^{*}\right)^{a-1} \hat{B}_{t} d \bar{W}_{t}\right)\right) .
$$

Proof. It follows that $V\left(r_{t}, t, T\right) \frac{1}{B_{t}}$ and $u\left(t, \hat{B}_{t}, T\right)$ are martingales under $P$. Furthermore, $W$ and $\bar{W}$ are independent, so that $\frac{V\left(r_{t}, t, T\right)}{B_{t}}$ and $u\left(t, \hat{B}_{t}, T\right)$ are independent. Hence we have by the Itto formula

$$
d(\hat{P}(t, T))=u\left(t, \hat{B}_{t}, T\right) d\left(\frac{V\left(r_{t}, t, T\right)}{B_{t}}\right)+\frac{V\left(r_{t}, t, T\right)}{B_{t}} d u\left(t, \hat{B}_{t}, T\right) .
$$

Again by the Itô-formula and the fact that $\frac{V\left(r_{t}, t, T\right)}{B_{t}}$ is a martingale it follows that

$$
d\left(\frac{V\left(r_{t}, t, T\right)}{B_{t}}\right)=\frac{1}{B_{t}} \frac{\partial V\left(r_{t}, t, T\right)}{\partial r_{t}} \sigma r_{t}^{3 / 2} d W_{t}
$$

We now again use the Itô-formula and the fact that $u\left(t, \hat{B}_{t}, T\right)$ is a martingale

$$
d\left(u\left(t, \hat{B}_{t}, T\right)\right)=\frac{\partial u\left(t, \hat{B}_{t}, T\right)}{\partial \hat{B}_{t}} d \hat{B}_{t}
$$

But

which completes the proof.

$$
d \hat{B}_{t}=d\left(\frac{1}{\bar{S}_{t}^{*}}\right)=-c \alpha_{t}^{1-a}\left(\bar{S}_{t}^{*}\right)^{a-1} \hat{B}_{t} d \bar{W}_{t}
$$

We now discuss the hedging of a given zero coupon bond

$$
P(t, T):=S_{t}^{*} E\left(\frac{1}{S_{T}^{*}} \mid \mathcal{A}_{t}\right) .
$$

From Corollary 7.1, we have

$$
d(\hat{P}(t, T))=a(t, T) d W_{t}+b(t, T) d \bar{W}_{t}
$$


where $a(\cdot, \cdot)$ and $b(\cdot, \cdot)$ are given in Corollary 7.1. We propose to hedge $P(t, T)$ with longer maturity using a zero coupon bond with shorter maturity

$$
P\left(t, T_{H}\right):=S_{t}^{*} E\left(\frac{1}{S_{T_{H}}^{*}} \mid \mathcal{A}_{t}\right)
$$

where $T_{H}<<T$. From Corollary 7.1, it follows that

$$
d\left(\hat{P}\left(t, T_{H}\right)\right)=a\left(t, T_{H}\right) d W_{t}+b\left(t, T_{H}\right) d \bar{W}_{t} .
$$

We invest $\delta_{t}^{H}$ units at time $t$ in $P\left(t, T_{H}\right)$, where

$$
\delta_{t}^{H} a\left(t, T_{H}\right)=a(t, T),
$$

i.e. we use $P\left(t, T_{H}\right)$ to eliminate the effect of $W$. Furthermore, we invest $\delta_{t}^{B}$ at time $t$ in the savings account, where

$$
\delta_{t}^{B}\left(-\theta_{t} \hat{B}_{t}\right)+\delta_{t}^{H} b\left(t, T_{H}\right)=b(t, T),
$$

so that $\delta^{B}$ eliminates the effect of $\bar{W}$. Finally, we use the GOP to make the portfolio self-financing, i.e.

$$
d P(t, T)=\delta_{t}^{H} d P\left(t, T_{H}\right)+\delta_{t}^{B} d B_{t}+\delta_{t}^{S} d S_{t}^{*} .
$$

The quantities $\delta_{t}^{H}$ and $\delta_{t}^{B}$ can be shown to satisfy the following equations

$$
\delta_{t}^{H}=\frac{a(t, T)}{a\left(t, T_{H}\right)}=\frac{\partial V\left(r_{t}, t, T\right)}{\partial r_{t}}\left(\frac{\partial V\left(r_{t}, t, T_{H}\right)}{\partial r_{t}}\right)^{-1} \frac{u\left(t, \hat{B}_{t}, T\right)}{u\left(t, \hat{B}_{t}, T_{H}\right)}
$$

and

$$
\delta_{t}^{B}=-\frac{a(t, T)}{a\left(t, T_{H}\right)} \frac{V\left(r_{t}, t, T_{H}\right)}{B_{t}} \frac{\partial u\left(t, \hat{B}_{t}, T\right)}{\partial \hat{B}_{t}}+\frac{V\left(r_{t}, t, T\right)}{B_{t}} \frac{\partial u\left(t, \hat{B}_{t}, T\right)}{\partial \hat{B}_{t}} .
$$

For computing $\delta_{t}^{H}$ and $\delta_{t}^{B}$, we find it useful to note that from equation (7.1) we get

$$
\begin{aligned}
& \frac{\partial u\left(t, \hat{B}_{t}, T\right)}{\partial \hat{B}_{t}} \\
& =\Psi\left(\lambda\left(t, \bar{S}_{t}^{*}\right), \frac{1}{1-a}, 0\right)-\left(\hat{B}_{t}\right)^{2 a-2} \frac{2(1-a)}{\Delta \varphi(t)} s\left(\lambda\left(t, \bar{S}_{t}^{*}\right), \frac{1}{1-a}, 0\right),
\end{aligned}
$$

where $s(\cdot, \nu, 0)$ is a probability density function of a $\chi_{\nu}^{2}(\lambda)$ non-central chi-squared distributed random variable with $\nu$ degrees of freedom and non-centrality parameter $\lambda$, see Baldeaux et al. (2014). We also have

$$
\begin{aligned}
& \frac{\partial V\left(r_{t}, t, T\right)}{\partial r_{t}} \\
= & \frac{\Gamma(\gamma-\alpha)}{\Gamma(\gamma)} \frac{\alpha}{r_{t}}\left(\frac{2}{\sigma^{2} r_{t} y(t, p)}\right)^{\alpha} \\
& \left(\frac{2}{\sigma^{2} \gamma r_{t} y(t, p)} M\left(\alpha+1, \gamma+1,-\frac{2}{\sigma^{2} r_{t} y(t, p)}\right)-M\left(\alpha, \gamma,-\frac{2}{\sigma^{2} r_{t} y(t, p)}\right)\right) .
\end{aligned}
$$

where the fact that $\frac{d M}{d z}(a, b, z)=\frac{a}{b} M(a+1, b+1, z)$ is used (Abramowitz and Stegun (1972)). 

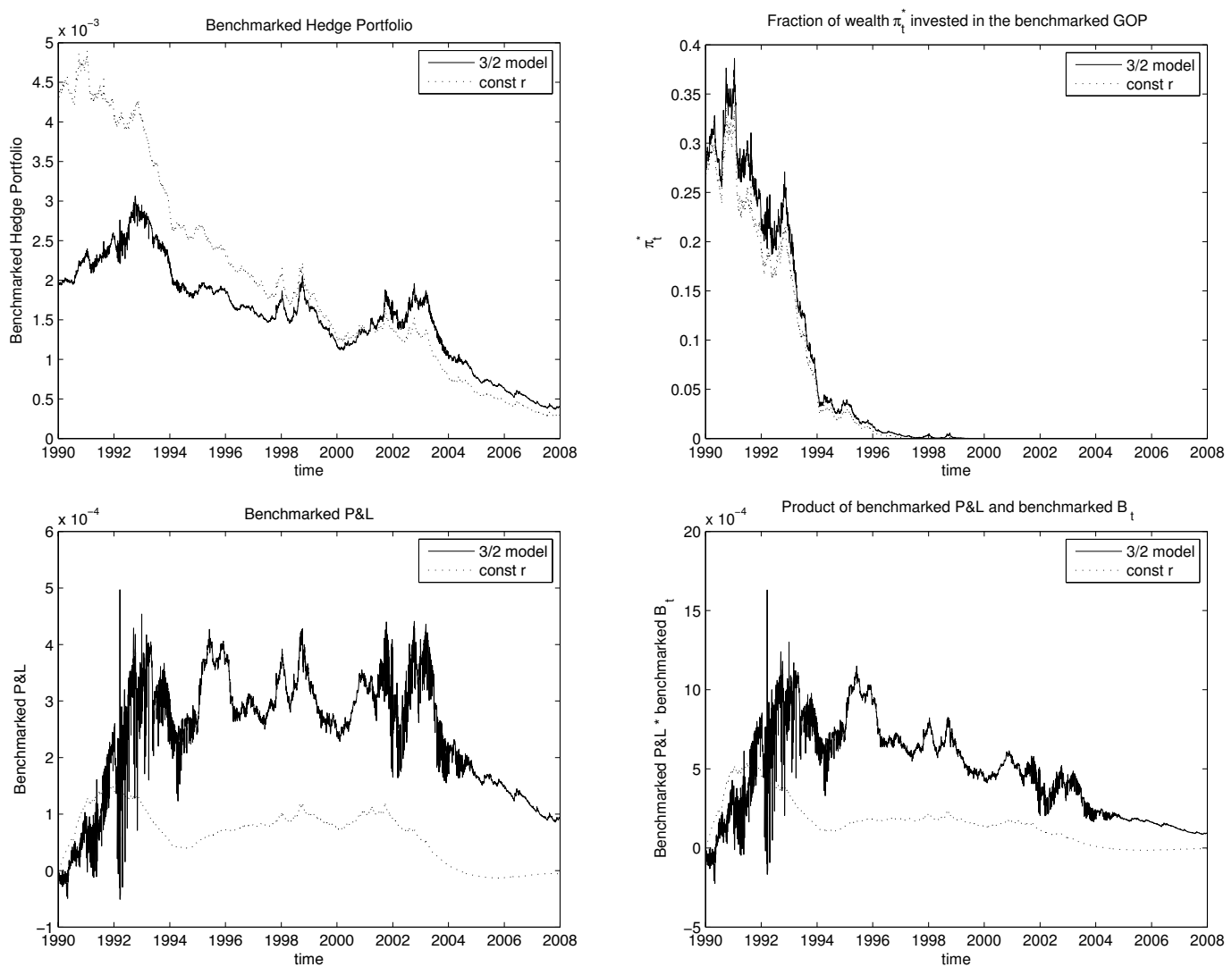

Fig. 8. Benchmarked hedge portfolio (upper left panel), estimated fraction of wealth invested for the GOP (upper right panel), the benchmarked profit and loss (lower left panel) in the hybrid model with stochastic interest rates as well as the TCEV model with constant interest rates and the product of the benchmarked profit and loss with the benchmarked savings account (lower right panel).

\subsection{Hedging Results}

In Section 6 we have identified the 3/2 model as the model which leads, in particular in the long term, to the lowest prices compared to the market. In this section we will rely on this model when hedging long dated zero coupon bonds by means of short-maturity zero coupon bonds, the savings account and the GOP. Since hedge results for other competing models appear to be very similar but lead to a more expensive hedge than the hybrid model using the $3 / 2$ short rate dynamics, we omit their discussion.

The zero coupon bond to be hedged has long maturity corresponding to about 18 years initially. The data, covering the time span from 01 January 1990 to 31 December 2008 are available on a daily basis from Bloomberg. The short maturity (one year) bonds are used as hedge instruments. Their prices are obtained from the corresponding swap rates ${ }^{7}$ using the Nelson-Siegel procedure, as described in Filipovic (2009). For these purposes, we employ 36 one-year bonds using each bond to cover 6 months: The first one-year bond corresponds to the time frame from 01 January 1990 to 31 December 1990, but we only use it for the initial six months period, i.e. from January 1990 to 30 June 1990. The second one-year bond corresponds to the time frame from 01 July 1990 to 30 June 1991, but we only use it for the initial six months, i.e. from 01 July 1990 to 31 December 1990, etc. ${ }^{8}$ As motivated above, since

\footnotetext{
7 Swap rates are available on a daily basis from Bloomberg.

8 This is done because for time to maturity less than six months, bond prices are close to unity, and do not exhibit enough variability to be useful for hedging.
} 
results presented in Section 7.1 assume that the hedging portfolio is rebalanced continuously, it seems to be relevant to employ daily data, rather than monthly data for hedging purposes. ${ }^{9}$

In the following, we show for the hybrid model with $3 / 2$ short rate dynamics and TCEV discounted GOP dynamics the resulting hedge performance. We compare the results with those for a model with constant interest rate and TCEV dynamics for the discounted GOP. Figure 8 shows the benchmarked hedge portfolio (left upper panel), the estimated fraction of wealth invested in the GOP (right upper panel) and the benchmarked profit and loss (P\&L) (lower panel) for the long maturity bond starting on 01 January 1990 and maturing in December 2007, under daily reallocation in the short-maturity bond, the savings account and the GOP. From the graph of the benchmarked hedge portfolio we observe that the model with constant interest rates produces a more expensive hedge than the hybrid model with stochastic interest rate. In fact, for the hybrid model with stochastic interest rates, the price turns out to be more than $50 \%$ lower than for the model with constant interest rates. Close to maturity, the portfolio with constant interest rates performs only slightly better. Furthermore, we observe that the underlying assumption regarding the benchmarked nonnegative portfolio to be a martingale, see Definition 5.1, seems to be better satisfied for the hybrid model, than for the model with constant interest rates. Note that all benchmarked nonnegative portfolios are supermartingales. The fraction of wealth $\pi_{t}^{*}$ invested in the EWI114, adjusted on a daily basis is shown in the right upper panel of Figure 8. The hybrid model suggests to invest a larger fraction of wealth in the GOP, compared to the model with constant interest rates. In this manner it exploits more the equity premium present in the index. Finally, when defining the benchmarked P\&L as the benchmarked gains from trade plus the initial benchmarked price minus the benchmarked price, the $3 / 2$ model with stochastic interest rates as well as the model with constant rates result in benchmarked P\&Ls that are both of comparable size and rather small. These are visualised in the lower left panel of Figure 8. The maximum absolute benchmarked P\&L amounts to less than 0.00005 for both model specifications. The lower right panel of Figure 8 displays the product of the benchmarked P\&L with the benchmarked savings account for both models. In both cases one does not observe any systematic trend. This suggests that there is no evidence that the benchmarked P\&L and its product with the benchmarked savings account could not be a local martingale, as requested by benchmarked risk minimization, see Du and Platen (2014).

\section{Conclusion}

This paper applies the benchmark approach (Platen and Heath (2010)) to pricing and hedging of long dated bonds, which are of crucial importance in the management of long term asset-liabilities in life insurance and annuity businesses. A hybrid approach to the modelling dynamics of the joint discounted growth optimal portfolio (GOP) and the short rate is introduced. It employs the real world pricing methodology of the benchmark approach under which the GOP is used as numéraire portfolio or benchmark and the real world probability measure as pricing measure. We compare a variety of tractable short rate models including the Vasicek, CIR, 3/2, Dothan and C\&L models. For modelling the discounted GOP we apply an extension of a time dependent constant elasticity of variance (TCEV) model. Using non-parametric kernel-based estimation we fit the dynamics of the discounted GOP and the short rate. It turns out that the power of the diffusion coefficient function for the short rate dynamics appears to be close to $3 / 2$, which makes the $3 / 2$ model more realistic than other models.

When applying the benchmark approach to pricing long dated zero coupon bonds of different maturities

9 Hedging performance has also been tested using diversified indices on a monthly basis, however, the daily hedging produces clearly better results. 
using different model specifications, we found that the market tends to be overpriced. That is, the model prices are generally lower than the respective market prices. Furthermore, we identify the $3 / 2$ short rate model as the best performing model in the sense that it is leading to the lowest bond prices compared to the market, as well as, the competing models. Finally, we demonstrate that the zero coupon bond payoff can be successfully hedged within the benchmark framework by using three instruments: a short-maturity zero coupon bond, the savings account and the GOP. In particular, the least expensive hedge was achieved by using the $3 / 2$ short rate model. It indicates that this hybrid model provides the most cost-efficient hedge. This model is highly tractable: It can be easily fitted and explicit formulas for bond prices and hedge ratios are available. Further research on the modelling, pricing and hedging under the benchmark approach will provide effective solutions to challenging risk management problems of long dated claims as they occur, for instance, for pension guarantees and variable annuities.

Table 4

Pricing error $\delta_{\tau}=\frac{P(t, T)^{i}-P(t, T)^{m}}{P(t, T)^{m}}$ computed for different model specifications (Vasicek, CIR, 3/2, Dothan and C\&L) and different maturities (6 month, 1 year, 5 years, 10 years, 20 years and 30 years). The results for the best performing model (the least expensive compared to the market) based on mean and median for each maturity are indicated in bold letters.

\begin{tabular}{|c|c|c|c|c|c|}
\hline$\overline{\text { Model }}$ & Vasicek & CIR & $3 / 2$ & Dothan & C\&L \\
\hline & \multicolumn{5}{|c|}{ 6-month maturity } \\
\hline mean & 0.00036 & 0.00036 & -0.00029 & 0.00067 & -0.00029 \\
\hline std.dev & 0.00094 & 0.00094 & 0.00103 & 0.00074 & 0.00077 \\
\hline median & 0.00036 & 0.00035 & -0.00023 & 0.00054 & -0.00039 \\
\hline $5 \%-Q$ & -0.00095 & -0.00096 & -0.00187 & -0.00027 & -0.00132 \\
\hline \multirow[t]{2}{*}{$95 \%-\mathrm{Q}$} & 0.00232 & 0.00231 & 0.00139 & 0.00236 & 0.00146 \\
\hline & \multicolumn{5}{|c|}{ 1-year maturity } \\
\hline mean & 0.00204 & 0.00200 & -0.00015 & 0.00316 & -0.00060 \\
\hline std.dev & 0.00351 & 0.00352 & 0.00316 & 0.00300 & 0.00302 \\
\hline median & 0.00220 & 0.00218 & -0.00015 & 0.00293 & -0.00075 \\
\hline $5 \%-Q$ & -0.00305 & -0.00312 & -0.00514 & -0.00123 & -0.00480 \\
\hline \multirow{2}{*}{$95 \%-\mathrm{Q}$} & 0.00882 & 0.00881 & 0.00539 & 0.00858 & 0.00455 \\
\hline & \multicolumn{5}{|c|}{ 5-years maturity } \\
\hline mean & 0.04908 & 0.04622 & -0.00797 & 0.07082 & -0.01113 \\
\hline std.dev & 0.04236 & 0.04251 & 0.05671 & 0.05088 & 0.04009 \\
\hline median & 0.03926 & 0.03628 & -0.01523 & 0.06923 & -0.01238 \\
\hline $5 \%-\mathrm{Q}$ & -0.00822 & -0.01004 & -0.09241 & -0.01066 & -0.07497 \\
\hline \multirow[t]{2}{*}{$95 \%-\mathrm{Q}$} & 0.13057 & 0.12900 & 0.08754 & 0.15315 & 0.05793 \\
\hline & \multicolumn{5}{|c|}{ 10-years maturity } \\
\hline mean & 0.16789 & 0.15438 & -0.04696 & 0.25182 & -0.02787 \\
\hline std.dev & 0.10811 & 0.10768 & 0.13730 & 0.15127 & 0.08886 \\
\hline median & 0.14526 & 0.13113 & -0.07378 & 0.22012 & -0.03565 \\
\hline $5 \%-Q$ & 0.03104 & 0.01781 & -0.22311 & 0.02458 & -0.16163 \\
\hline \multirow[t]{2}{*}{$95 \%-\mathrm{Q}$} & 0.37994 & 0.36598 & 0.25032 & 0.48341 & 0.13947 \\
\hline & \multicolumn{5}{|c|}{ 20-years maturity } \\
\hline mean & 0.23024 & 0.1863 & -0.34269 & 0.56986 & -0.18914 \\
\hline std.dev & 0.25683 & 0.2502 & 0.14513 & 0.36089 & 0.16616 \\
\hline median & 0.16296 & 0.1194 & -0.36058 & 0.50857 & -0.22827 \\
\hline $5 \%-Q$ & -0.07341 & -0.1075 & -0.54098 & 0.04172 & -0.39284 \\
\hline \multirow{2}{*}{$95 \%-\mathrm{Q}$} & 0.77952 & 0.7224 & -0.10517 & 1.15016 & 0.16426 \\
\hline & \multicolumn{5}{|c|}{ 30-years maturity } \\
\hline mean & -0.38529 & -0.42268 & -0.79296 & 0.00336 & -0.64277 \\
\hline std.dev & 0.21099 & 0.19978 & 0.06356 & 0.31659 & 0.12500 \\
\hline median & -0.45070 & -0.48261 & -0.80518 & -0.04761 & -0.67854 \\
\hline $5 \%-Q$ & -0.61573 & -0.63849 & -0.87569 & -0.43931 & -0.77820 \\
\hline $95 \%-\mathrm{Q}$ & 0.06737 & 0.00777 & -0.66829 & 0.59847 & -0.36915 \\
\hline
\end{tabular}



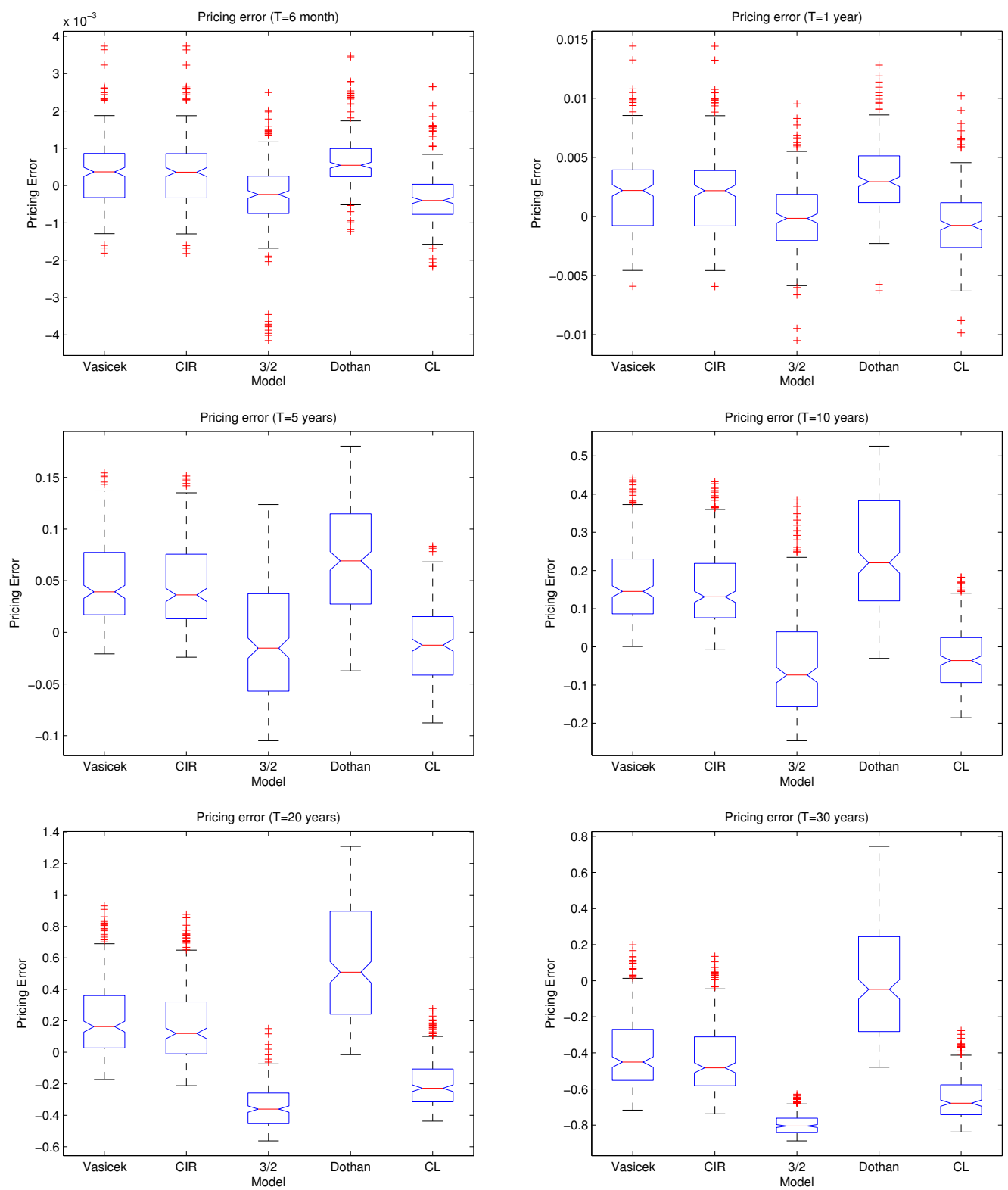

Fig. 9. Box-plots for the in-sample pricing errors $\delta_{\tau}=\frac{P(t, T)^{i}-P(t, T)^{m}}{P(t, T)^{m}}$ for zero coupon bonds computed for different model specifications (Vasicek, CIR, 3/2, Dothan and C\&L) and different maturities (6 month, 1 year, 5 years, 10 years, 20 years and 30 years). 


\section{References}

Abramowitz, M., Stegun, I. A. e., 1972. Handbook of Mathematical Functions with Formulas, Graphs, and Mathematical Tables. Dover, New York.

Ahn, D. H., Gao, B., 1999. A Parametric Nonlinear Model of Term Structure Dynamics. Review of Financial Studies 12, 721-762.

Baldeaux, J., Ignatieva, K., Platen, E., 2014. A Tractable Model for Indices Approximating the Growth Optimal Portfolio. Studies in Nonlinear Dynamics and Econometrics 18(1), 1-21.

Black, F., Derman, E., Toy, W., 1990. A One-Factor Model of Interest Rates and Its Application to Treasury Bond Options. Financial Analysts Journal 46(1), 33-39.

Botev, Z., Grotowski, J. F., Kroese, D. P., 2010. Kernel Density Estimation via Diffusion. The Annals of Statistics 38(5), 2916-2957.

Breiman, L., 1960. Investment Policies for Expanding Business Optimal in a Long Run Sense. Naval Research Logistics Quarterly 7 (4), 647-651.

Brennan, M. J., Schwartz, E. S., 1977. Savings Bonds, Retractable Bonds, and Callable Bonds. Journal of Financial Economics 5, 67-88.

Brennan, M. J., Schwartz, E. S., 1979. A Continuous Time Approach to the Pricing of Bonds. Journal of Banking and Finance 3, 133-155.

Brennan, M. J., Schwartz, E. S., 1980. Analyzing Convertible Bonds. Journal of Financial and Quantitative Analysis 15, 907-929.

Brigo, D., Mercurio, F., 2001. Interest Rate Models - Theory and Practice, 1st Edition. Springer.

Buchwalder, M., Bühlmann, H., Merz, M., Wüthrich, M. V., 2007. Valuation Portfolio in Non-Life Insurance. Scandinavian Actuarial Journal 2007(2), 108-125.

Carr, P., Sun, J., 2007. A New Approach for Option Pricing under Stochastic Volatility. Review of Derivatives Research 10, 87-150.

Ceci, C., Colaneri, K., Cretarola, A., 2014. A Benchmark Approach to Risk-Minimization under Partial Information. Insurance: Mathematics \& Economics 55, 129-146.

Chan, K. C., Karolyi, G. A., Longstaff, F. A., Sanders, A., 1992. An Empirical Comparison of Alternative Models of the Short-Term Interest Rate. The Journal of Finance 47(3), 1209-1227.

Cox, J., 1996. The Constant Elasticity of Variance Option Pricing Model. Journal of Portfolio Management 22 (special issue), 15-17.

Cox, J., Ingersoll, J., Ross, S., 1985. A Theory of the Term Structure of Interest Rates. Econometrica 53, 385-407.

Craddock, M., Lennox, K., 2007. Lie Group Symmetries as Integral Transforms of Fundamental Solutions. Journal of Differential Equations 232, 652-674.

Dothan, L. U., 1978. On the Term Structure of Interest. Journal of Financial Economics 6, 59-69.

Du, K., Platen, E., 2014. Benchmarked Risk Minimization. To appear in Mathematical Finance.

Epanechnikov, V., 1969. Nonparametric Estimates of Multivariate Probability Density. Theory of Probability and Applications 14, 153-158.

Filipovic, D., 2009. Term-Structure Models: A Graduate Course. Springer-Verlag.

Florens-Zmirou, D., 1993. On Estimating the Diffusion Coefficient from Discrete Observations. Journal of Applied Probability 30(4), 790-804.

Gibson, R., Schwartz, E. S., 1990. Stochastic Convenience Yield and the Pricing of Oil Contingent Claims. Journal of Finance 45, 959-976.

Hull, J., White, A., 1992. Pricing Interest-Rate Derivative Securities. Review of Financial Studies 3, $573-592$.

Ignatieva, K., Platen, E., 2012. Estimating the Diffusion Coefficient Function for a Diversified World Stock Index. Computational Statistics \& Data Analysis 56(6), 1333-1349.

Jacod, J., 2000. Non-Parametric Kernel Estimation of the Coeffcient of a Diffusion. Scandinavian Journal of Statistics 27(1), 83-96.

Jamshidian, F., 1990. An Exact Bond Option Formula. Journal of Finance 44, 205-209. 
Jeanblanc, M., Yor, M., Chesney, M., 2009. Mathematical Methods for Financial Markets. Springer.

Jiang, G., Knight, J., 1997. A Nonparametric Approach to the Estimation of Diffusion Processes, with an Application to a Short-Term Interest Rate Model. Econometric Theory 13(7), 615-645.

Karatzas, I., Shreve, S. E., 1991. Brownian Motion and Stochastic Calculus, 2nd Edition. Springer.

Kelly, J. R., 1956. A New Interpretation of Information Rate. Bell Syst. Techn. J. 35, 917-926.

Künsch, H. R., 1989. The Jackknife and the Bootstrap for General Stationary Observations. The Annals of Statistics 17, 1217-1241.

Latané, H., 1959. Criteria for Choice among Risky Ventures. Journal of Political Economy 38, 145-155.

Lim, A., Wong, B., 2010. A Benchmarking Approach to Optimal Asset Allocation for Insurers and Pension Funds. Insurance: Mathematics \& Economics 46, 317-327.

Long, J. B., 1990. The Numeraire Portfolio. Journal of Financial Economics 26, 29-69.

Longstaff, F. A., 1989. A Nonlinear General Equilibrium Model of the Term Structure of Interest Rates. Journal of Financial Economics 23, 195-224.

Longstaff, F. A., 1990. The Valuation of Options on Yields. Journal of Financial Economics 26, 97-122.

Longstaff, F. A., Schwartz, E. S., 1992. Interest-Rate Volatility and the Term Structure: A Two-Factor General Equilibrium Model. Journal of Finance 47(4), 1259-1282.

Markowitz, H., 1976. Investment for the Long Run: New Evidence for an Old Rule. Journal of Finance 31 (5), 1273-1286.

Merton, R., 1973. Theory of Rational Option Pricing. Bell Journal of Economics and Management Science 4, 141-183.

Miller, S., Platen, E., 2005. A Two-Factor Model for Low Interest Rate Regimes. Asia-Pacific Financial Markets 11, 107-133.

Musiela, M., Rutkowski, M., 2005. Martingale Methods in Financial Modelling, 2nd Edition. Vol. 36. Springer.

Nowak, P., Romaniuk, M., 2013. Pricing and Simulations of Catastrophe Bonds. Insurance: Mathematics \& Economics 52(1), 18-28.

Peng, J., Leung, K. S., Kuen, Y., 2012. Pricing Guaranteed Minimum Withdrawal Benefits under Stochastic Interest Rates. Quantitative Finance 12(6), 933-941.

Pintoux, C., Privault, N., 2011. The Dothan Pricing Model Revisited. Mathematical Finance 21, 355363.

Platen, E., 1999. A Short Term Interest Rate Model. Finance and Stochastics 3(2), 215-225.

Platen, E., Bruti-Liberati, N., 2010. Numerical Solution of Stochastic Differential Equations with Jumps in Finance. Springer-Verlag.

Platen, E., Heath, D., 2010. A Benchmark Approach to Quantitative Finance. Springer-Verlag.

Platen, E., Rendek, R., 2012. Approximating the Numeraire Portfolio by Naive Diversification. The Journal of Asset Management 31(1), 34-50.

Ramaswamy, K., Sundaresan, S. M., 1986. The Valuation of Floating-Rate Instruments. Journal of Financial Economics 17, 251-272.

Rebonato, R., 1998. Interest-Rate Option Models, 2nd Edition. Wiley Series in Financial Engineering, John Wiley and Sons.

Silverman, B. W., 1986. Density Estimation. Chapman and Hall.

Soulier, P., 1998. Nonparametric Estimation of the Diffusion Coefficient of a Diffusion Process. Stochastic Analysis and Applications 16, 185-200.

Stanton, R., 1997. A Nonparametric Model of Term Structure Dynamics and the Market Price of Interest Rate Risk. Journal of Finance 52(5), 1973-2002.

Thorp, E. O., 1961. A Favourable Strategy for Twenty-One. In: Proceedings of the National Academy of Sciences. Vol. 47. pp. 110-112.

Vasicek, O., 1977. An Equilibrium Characterisation of the Term Structure. Journal of Financial Economics 5, 177-188.

Wand, M. P., Jones, M. C., 1995. Kernel Smoothing. Chapman and Hall. 